Can. J. Math. Vol. 49 (2), 1997 pp. 193-211

\title{
CLASSIFYING PL 5-MANIFOLDS BY REGULAR GENUS: THE BOUNDARY CASE
}

\author{
MARIA RITA CASALI
}

\begin{abstract}
In the present paper, we face the problem of classifying classes of orientable PL 5-manifolds $M^{5}$ with $h \geq 1$ boundary components, by making use of a combinatorial invariant called regular genus $\mathcal{G}\left(M^{5}\right)$. In particular, a complete classifi-
\end{abstract} cation up to regular genus five is obtained:

$$
\mathcal{G}\left(M^{5}\right)=\varrho \leq 5 \Longrightarrow M^{5} \cong \#_{\varrho^{-}-\varrho}\left(\mathbb{S}^{4} \times \mathbb{S}^{1}\right) \#^{(h)} \mathbb{W}_{\mathbb{\rho}},
$$

where ${ }^{\partial} \varrho=\mathcal{G}\left(\partial M^{5}\right)$ denotes the regular genus of the boundary $\partial M^{5}$ and ${ }^{(h)} \mathbb{H}_{\partial_{\varrho}}$ denotes the connected sum of $h \geq 1$ orientable 5-dimensional handlebodies $\mho_{\alpha_{i}}$ of genus $\alpha_{i} \geq 0$ $(i=1, \ldots, h)$, so that $\sum_{i=1}^{h} \alpha_{i}={ }^{\partial}$.

Moreover, we give the following characterizations of orientable PL 5-manifolds $M^{5}$ with boundary satisfying particular conditions related to the "gap" between $\mathcal{G}\left(M^{5}\right)$ and either $\mathcal{G}\left(\partial M^{5}\right)$ or the rank of their fundamental group $\operatorname{rk}\left(\pi_{1}\left(M^{5}\right)\right)$ :

$$
\begin{gathered}
\mathcal{G}\left(\partial M^{5}\right)=\mathcal{G}\left(M^{5}\right)=\varrho \Longleftrightarrow M^{5} \cong\left({ }^{(h)} \mathbb{H}_{\varrho}\right. \\
\mathcal{G}\left(\partial M^{5}\right)={ }^{\partial} \varrho=\mathcal{G}\left(M^{5}\right)-1 \Longleftrightarrow M^{5} \cong\left(\mathbb{S}^{4} \times \mathbb{S}^{1}\right) \#^{(h)} \mathbb{H}_{\partial_{\varrho}} \\
\mathcal{G}\left(\partial M^{5}\right)=\partial_{\varrho}=\mathcal{G}\left(M^{5}\right)-2 \Longleftrightarrow M^{5} \cong \#_{2}\left(\mathbb{S}^{4} \times \mathbb{S}^{1}\right) \#^{(h)} \mathbb{H}_{\partial_{\varrho}} \\
\mathcal{G}\left(M^{5}\right)=\operatorname{rk}\left(\pi_{1}\left(M^{5}\right)\right)=\varrho \Longleftrightarrow M^{5} \cong \#_{\varrho-{ }^{2}}\left(\mathbb{S}^{4} \times \mathbb{S}^{1}\right) \#^{(h)} \mathbb{W}_{\partial_{\varrho}} .
\end{gathered}
$$

Further, the paper explains how the above results (together with other known properties of regular genus of PL manifolds) may lead to a combinatorial approach to 3dimensional Poincaré Conjecture.

1. Introduction. As far as closed orientable PL 5-manifolds are concerned, many (partial) classification results are known, which make use of a (non-negative) combinatorial invariant called regular genus (see $\left[\mathrm{G}_{1}\right]$ ); in particular, we may collect them into the following statement, where the symbol $\cong$ means PL-homeomorphism, $\#_{m}\left(\mathbb{S}^{4} \times \mathbb{S}^{1}\right)$ denotes the connected sum of $m$ copies of $\mathbb{S}^{4} \times \mathbb{S}^{1}$, and $\mathbb{S}^{3} \times \mathbb{S}^{2}\left(\right.$ resp. $\left.\mathbb{S}^{3} \underset{\sim}{\times} \mathbb{S}^{2}\right)$ indicates the trivial (resp. non trivial) 3 -sphere bundle over the 2 -sphere.

THEOREM 1 ([FG 1$\left.],[\mathrm{CG}],\left[\mathrm{C}_{3}\right]\right)$. Let $M^{5}$ be a closed orientable PL 5-manifold, with regular genus $\mathcal{G}\left(M^{5}\right)$, and fundamental group of rank $\operatorname{rk}\left(\pi_{1}\left(M^{5}\right)\right)$.

a) $\mathcal{G}\left(M^{5}\right)=0 \Leftrightarrow M^{5} \cong \mathbb{S}^{5}$;

Work performed under the auspicies of the G.N.S.A.G.A. of the C.N.R. (National Research Council of Italy) and financially supported by M.U.R.S.T. of Italy (project "Topologia e Geometria").

Received by the editors November 16, 1995; revised February 22, 1996.

AMS subject classification: Primary: 57N15, 57Q15; Secondary: 05C10.

(c) Canadian Mathematical Society 1997. 
b) $\mathcal{G}\left(M^{5}\right)=m \leq 7 \Leftrightarrow M^{5} \cong \#_{m}\left(\mathbb{S}^{4} \times \mathbb{S}^{1}\right)$;

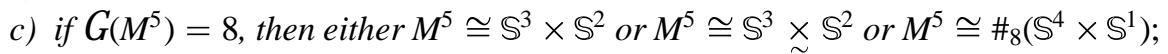

d) $\mathcal{G}\left(M^{5}\right)=\operatorname{rk}\left(\pi_{1}\left(M^{5}\right)\right)=m \Leftrightarrow M^{5} \cong \#_{m}\left(\mathbb{S}^{4} \times \mathbb{S}^{1}\right)$;

e) it is impossible that $\mathrm{rk}\left(\pi_{1}\left(M^{5}\right)\right)+1 \leq \mathcal{G}\left(M^{5}\right) \leq \mathrm{rk}\left(\pi_{1}\left(M^{5}\right)\right)+7$;

f) if $\mathcal{G}\left(M^{5}\right)=m=\operatorname{rk}\left(\pi_{1}\left(M^{5}\right)\right)+8$ and $\pi_{1}\left(M^{5}\right)$ is a free group, then either $M^{5} \cong$

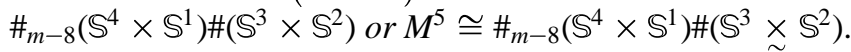

In the present paper, the attention is fixed upon orientable PL 5-manifolds with boundary, and the classification effort is carried out in case of "low" regular genus, in case of "restricted gap" between the regular genus of the manifold and the regular genus of its boundary ${ }^{1}$, and in case of "restricted gap" between the regular genus and the rank of the fundamental group of the manifold.

The main results achieved are presented in the following theorems, where $M^{5}$ is assumed to be an orientable PL 5-manifold with $h \geq 1$ boundary components, $\mathbb{S}^{2} \times \mathbb{D}^{3}$ (resp. $\mathbb{S}^{2} \underset{\sim}{\times} \mathbb{D}^{3}$ ) indicates the trivial (resp. nontrivial) 3-ball bundle over the 2-sphere, and ${ }^{2} \#$ denotes the boundary connected sum; further, if $\Upsilon_{\alpha}(\alpha \geq 0)$ denotes the orientable 5dimensional handlebody of genus $\alpha$ (i.e. the orientable PL 5-manifold obtained from the 5-ball $\mathbb{D}^{5}$ by pairwise identification of $2 \alpha$ disjoint 4-balls of its boundary), then we will always indicate by ${ }^{(h)} \mathbb{t}_{\alpha}$ the connected sum ${ }^{(h)} \mathbb{t}_{\alpha}=\mathbb{Y}_{\alpha_{1}} \# \cdots \# \mathbb{Y}_{\alpha_{h}}$, with $\sum_{i=1}^{h} \alpha_{i}=\alpha$.

THEOREM 2. If $\mathcal{G}\left(M^{5}\right)=\varrho \leq 5$, then $M^{5} \cong \#_{\varrho-\chi}\left(\mathbb{S}^{4} \times \mathbb{S}^{1}\right) \#^{(h)} \mathbb{Z}_{\text {}}$, where $\partial_{\varrho}=$ $\mathcal{G}\left(\partial M^{5}\right)\left(0 \leq \partial^{\partial} \leq \varrho\right)$.

THEOREM 3. a) $\mathcal{G}\left(\partial M^{5}\right)=\mathcal{G}\left(M^{5}\right)=\varrho \Leftrightarrow M^{5} \cong{ }^{(h)} \mathbb{H}_{\varrho}$;

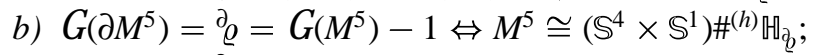

c) $\mathcal{G}\left(\partial M^{5}\right)=\partial_{\varrho}=\mathcal{G}\left(M^{5}\right)-2 \Leftrightarrow M^{5} \cong \#_{2}\left(\mathbb{S}^{4} \times \mathbb{S}^{1}\right) \#^{(h)} \mathbb{W}_{z_{2}}$;

d) if $\mathcal{G}\left(\partial M^{5}\right)=\partial_{\varrho}=\mathcal{G}\left(M^{5}\right)-3$ and $\pi_{1}\left(M^{5}\right)=*_{m} \mathbb{Z}$ then either $M^{5} \cong$

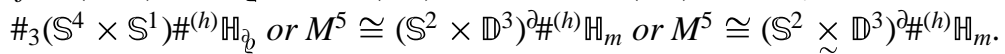

THEOREM 4. a) $\mathcal{G}\left(M^{5}\right)=\operatorname{rk}\left(\pi_{1}\left(M^{5}\right)\right)=\varrho \Leftrightarrow M^{5} \cong \#_{\varrho_{-}(}\left(\mathbb{S}^{4} \times \mathbb{S}^{1}\right) \#^{(h)} \mathbb{Z}_{q_{2}}$, where $\partial_{\varrho}=\mathcal{G}\left(\partial M^{5}\right)$;

b) it is impossible that $\operatorname{rk}\left(\pi_{1}\left(M^{5}\right)\right)+1 \leq \mathcal{G}\left(M^{5}\right) \leq \operatorname{rk}\left(\pi_{1}\left(M^{5}\right)\right)+4$;

c) if $M^{5} \neq \#_{\varrho-\chi_{e}}\left(\mathbb{S}^{4} \times \mathbb{S}^{1}\right) \#^{(h)} \mathbb{H}_{\text {z }}$, then $\mathcal{G}\left(M^{5}\right) \geq \operatorname{rk}\left(\pi_{1}\left(M^{5}\right)\right)+5+\beta_{2}\left(\partial M^{5}\right)$, where $\beta_{2}\left(\partial M^{5}\right)$ denotes the second Betti number of the boundary $\partial M^{5}$;

d) if $\mathcal{G}\left(M^{5}\right)=\operatorname{rk}\left(\pi_{1}\left(M^{5}\right)\right)+5$, then $\partial M^{5} \cong \amalg\left\{\#_{\alpha_{i}}\left(\mathbb{S}^{3} \times \mathbb{S}^{1}\right) / i=1, \ldots, h\right\}$, where $\amalg$ denotes a disjoint union and $\sum_{i=1}^{h} \alpha_{i}={ }^{\partial}$.

The proof of Theorems 2, 3 and 4 will be postponed till Section 4; in fact, the paper is organized as follows:

- in Section 2, we introduce and analyze the so called $(1, n-1)$-handle presentations of PL $n$-manifolds, for $n \geq 4$ (i.e. particular handle-presentations, where only handles of indices 1 and $n-1$ appear);

1 Note that—as proved in [CP]—the inequality $\mathcal{G}\left(\partial M^{n}\right) \leq \mathcal{G}\left(M^{n}\right)$ holds for every PL $n$-manifold $M^{n}$. 
- in Section 3, we give the necessary preliminaries about regular genus and its background (i.e. the representation theory of PL manifolds by edge-coloured graphs); further, some technical Lemmas are obtained, yielding the combinatorial hypothesis which ensure a 5-manifold $M^{5}$ to admit a $(1, n-1)$-handle presentation;

- in Section 4, the main theorems are proved, together with other consequences (see, for example, Corollary 9 and Corollary 10 , where $\mathcal{G}\left(\mathbb{S}^{2} \times \mathbb{D}^{3}\right)=7$ and $\mathcal{G}\left(\mathbb{S}^{3} \times \mathbb{D}^{2}\right)=6$ are respectively computed).

Moreover, we note that the results of the present paper, arranged with other known properties of the regular genus, lead to a possible combinatorial approach to 3-dimensional Poincaré Conjecture.

In [CG], the following sequence of conjectures appears, and Conjectures $\mathrm{C}_{1}$ and $\mathrm{C}_{2}$ together are shown to imply Poincaré Conjecture, for all closed orientable 3-manifolds $M^{3}$ of Heegaard genus $\mathcal{H}\left(M^{3}\right) \leq 5$.

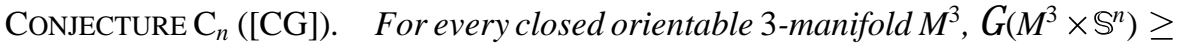
$\mathcal{G}\left(M^{3} \times \mathbb{D}^{n}\right)$.

Now, we are able to prove that Conjectures $\mathrm{C}_{1}$ and $\mathrm{C}_{2}$ together really imply Poincaré Conjecture, for every closed orientable 3-manifold.

In fact, by Conjecture $\mathrm{C}_{2}$ and Theorem $3, \mathcal{G}\left(M^{3} \times \mathbb{S}^{2}\right) \geq \mathcal{G}\left(M^{3} \times \mathbb{D}^{2}\right) \geq \mathcal{G}\left(M^{3} \times \mathbb{S}^{1}\right)+3$ follows; on the other hand, $\mathcal{G}\left(M^{3} \times \mathbb{D}^{1}\right) \geq \mathcal{G}\left(\partial\left(M^{3} \times \mathbb{D}^{1}\right)\right)+2=2 \mathcal{G}\left(M^{3}\right)+2$ is a direct consequence of [CM; Proposition 5] (where arbitrary 4-manifolds $M^{4}$ with $\mathcal{G}\left(M^{4}\right)$ $\mathcal{G}\left(\partial M^{4}\right) \leq 1$ are proved to be decomposable as connected sums of 4-dimensional handlebodies and—possibly—of $\mathbb{S}^{3} \times \mathbb{S}^{1}$ ). Thus, by assuming Conjecture $\mathrm{C}_{1}$, we have

$$
\mathcal{G}\left(M^{3} \times \mathbb{S}^{2}\right) \geq \mathcal{G}\left(M^{3} \times \mathbb{S}^{1}\right)+3 \geq \mathcal{G}\left(M^{3} \times \mathbb{D}^{1}\right)+3 \geq 2 \mathcal{G}\left(M^{3}\right)+5 .
$$

Poincaré Conjecture now easily would follow, since [CG; Corollary I] and [B] imply that $\mathcal{G}\left(\Sigma^{3} \times \mathbb{S}^{2}\right)=8$, for every homotopy 3 -sphere $\Sigma^{3}$, and since $\mathcal{G}\left(\Sigma^{3}\right)=\mathcal{H}\left(\Sigma^{3}\right) \leq 1$ is an obvious contradiction.

2. PL $n$-manifolds admitting (1,n-1)-handle presentations. It is well-known that every PL $n$-manifold with boundary ${ }^{2}$ admits a handle-presentation of type

$$
M^{n}=H^{(0)} \cup\left(H_{1}^{(1)} \cup \cdots \cup H_{r_{1}}^{(1)}\right) \cup \cdots \cup\left(H_{1}^{(n-1)} \cup \cdots \cup H_{r_{n-1}}^{(n-1)}\right)
$$

where every $p$-handle $H_{i}^{(p)}=\mathbb{D}^{p} \times \mathbb{D}^{n-p}\left(1 \leq p \leq n-1,1 \leq i \leq r_{p}\right)$ is endowed with an embedding (called attaching map) $f_{i}^{(p)}: \partial \mathbb{D}^{p} \times \mathbb{D}^{n-p} \rightarrow \partial\left(H^{(0)} \cup \cdots \cup\left(H_{1}^{(p-1)} \cup \cdots \cup H_{r_{p-1}}^{(p-1)}\right)\right)$.

DEFINITION 1. A $(1, n-1)$-handle presentation of a PL $n$-manifold $M^{n}$ (with $\partial M^{n} \neq$ $\emptyset)$ is a handle-presentation where only 1-handles and $(n-1)$-handles appear, i.e.

$$
M^{n}=H^{(0)} \cup\left(H_{1}^{(1)} \cup \cdots \cup H_{r}^{(1)}\right) \cup\left(H_{1}^{(n-1)} \cup \cdots \cup H_{s}^{(n-1)}\right) \quad(0 \leq s \leq r) .
$$

\footnotetext{
2 For all basic notions of piecewise-linear (PL) topology, we refer to [RS].
} 
The purpose of the present section is to analyze the topological structure of PL $n$ manifolds admitting a $(1, n-1)$-handle presentation, for $n \geq 5$; note that, in dimension $n=3$, the class of $(1, n-1)$-handle presentations coincides with the whole class of handle-presentations for manifolds with boundary, while in dimension $n=4$ the same problem has already been faced and solved in [CM, Theorem 1].

The following—quite obvious—statement will be helpful for our research; here, $\Upsilon_{\alpha}^{n}$ denotes the $n$-dimensional handlebody of genus $\alpha \geq 0$.

PROPOSITION 1. A PL n-manifold $M^{n}$, with $h \geq 1$ boundary components, admits a $(1, n-1)$-handle presentation if and only if it admits a decomposition of type

$$
M^{n}=\mathbb{Y}_{r}^{n} \cup_{\varphi} \mathbb{Y}_{s}^{n} \quad(0 \leq s \leq r)
$$

where $\varphi: \partial Y_{s}^{n}-\bigcup_{i=1}^{h} \operatorname{int}\left(\mathbb{D}_{(i)}^{n-1}\right) \rightarrow \partial \Upsilon_{r}^{n}$ is a regular embedding and $\mathbb{D}_{(1)}^{n-1}, \ldots, \mathbb{D}_{(h)}^{n-1}$ are $h$ disjoint $(n-1)$-balls contained into $\partial \mho_{s}^{n}$.

Now, we are able to prove the characterization theorem for $n$-manifolds $(n \geq 5$ ) admitting a $(1, n-1)$-handle presentation; here, ${ }^{(h)} \mathbb{E}_{\alpha}^{n}$ denotes the connected sum of $h \geq 1$ $n$-dimensional handlebodies $\mho_{\alpha_{i}}^{n}$ of genus $\alpha_{i} \geq 0(i=1, \ldots, h)$, so that $\sum_{i=1}^{h} \alpha_{i}=\alpha$.

PROPOSITION 2. For every $n \geq 5$, a PL n-manifold $M^{n}$, with $h \geq 1$ boundary components, admits $a(1, n-1)$-handle presentation (with $r \geq 01$-handles and $s \leq r(n-1)$ handles) if and only if

$$
M^{n} \cong \#_{s}\left(\mathbb{S}^{n-1} \times \mathbb{S}^{1}\right) \#^{(h)} \mathbb{\boxplus}_{r-s}^{n} .
$$

PROOF. Via Proposition 1, one implication is just an obvious remark: $\#_{s}\left(\mathbb{S}^{n-1} \times \mathbb{S}^{1}\right) \#^{(h)} \mathbb{Q}_{r-s}^{n}$ may be decomposed as $\mathbb{Y}_{r}^{n} \cup_{\bar{\varphi}} \mathbb{Y}_{s}^{n}(0 \leq s \leq r)$, where $\bar{\varphi}: \partial \mathbb{Y}_{s}^{n}-$ $\bigcup_{i=1}^{h} \operatorname{int}\left(\mathbb{D}_{(i)}^{n-1}\right) \rightarrow \partial \mathbb{Y}_{r}^{n}$ is the canonical embedding which sends the $i$-th $\mathbb{S}^{1}$-factor of $\partial \mathbb{Y}_{s}^{n}=\#_{s}\left(\mathbb{S}^{n-2} \times \mathbb{S}^{1}\right)$ into the $i$-th $\mathbb{S}^{1}$-factor of $\partial \mathbb{Y}_{r}^{n}=\#_{r}\left(\mathbb{S}^{n-2} \times \mathbb{S}^{1}\right)$, for every $1 \leq i \leq s$.

As far as the other implication is concerned, let us consider $M^{n}=\mathbb{V}_{r}^{n} \cup_{\varphi} \mathbb{\mho}_{s}^{n}, \varphi: \partial \mathbb{Y}_{s}^{n}-$ $\bigcup_{i=1}^{h} \operatorname{int}\left(\mathbb{D}_{(i)}^{n-1}\right) \rightarrow \partial \mho_{r}^{n}$ being a given regular embedding.

Now, if $\mathbb{S}_{(i)}^{1}, 1 \leq i \leq s$, is the $i$-th $\mathbb{S}^{1}$-factor of $\partial \mathbb{Y}_{s}^{n}=\#_{s}\left(\mathbb{S}^{n-2} \times \mathbb{S}^{1}\right)$, then the stuple $\left(\mathbb{S}_{(1)}^{\overline{1}}=\varphi\left(\mathbb{S}_{(1)}^{1}\right), \ldots, \mathbb{S}_{(s)}^{\overline{1}}=\varphi\left(\mathbb{S}_{(s)}^{1}\right)\right)$ may be completed to an r-tuple $\left(\mathbb{S}_{(1)}^{\overline{1}}, \ldots, \mathbb{S}_{(s)}^{\overline{1}}\right.$, $\left.\mathbb{S}_{(s+1)}^{\overline{1}}, \ldots, \mathbb{S}_{(r)}^{\overline{1}}\right)$ of generators for the fundamental group of $\partial \mathbb{Y}_{r}^{n}=\#_{r}\left(\mathbb{S}^{n-2} \times \mathbb{S}^{1}\right)$. By [L] and [LP], a self-homeomorphism $\psi$ of $\partial \mathbb{\mho}_{r}^{n}$ exists, so that $\psi\left(\mathbb{S}_{(i)}^{\mathrm{I}}\right)$ is the $i$-th $\mathbb{S}^{1}$-factor of $\partial \mathbb{Y}_{r}^{n}=\#_{r}\left(\mathbb{S}^{n-2} \times \mathbb{S}^{1}\right)$, for every $1 \leq i \leq r$, and so that $\psi$ may be extended to a selfhomeomorphism $\tilde{\psi}$ of the whole handlebody $\mathbb{Y}_{r}^{n}{ }^{3}{ }^{3}$ Moreover, [LP], [M] and [CH] state that any self-homeomorphism $f: \#_{p}\left(\mathbb{S}^{m-1} \times \mathbb{S}^{1}\right) \rightarrow \#_{p}\left(\mathbb{S}^{m-1} \times \mathbb{S}^{1}\right),(m \geq 3, p \geq 0)$ extends to a self-homeomorphism $\tilde{f}: \mathbb{Y}_{p}^{m+1} \rightarrow \mathbb{Y}_{p}^{m+1}$. Thus, the composition $\bar{\varphi}^{-1} \circ \psi \circ \varphi: \partial \mathbb{Y}_{s}^{n}-$ $\bigcup_{i=1}^{h} \operatorname{int}\left(\mathbb{D}_{(i)}^{n-1}\right) \longrightarrow \partial \mho_{s}^{n}-\bigcup_{i=1}^{h} \operatorname{int}\left(\mathbb{D}_{(i)}^{n-1}\right)$ may be first completed to a self-homeomorphism

\footnotetext{
3 Note that, by $[\mathrm{L}]$ and $[\mathrm{LP}], \psi$ is generated by sliding 1-handles, twisting 1-handles, permuting 1-handles and rotations.
} 
$T: \partial \mho_{s}^{n} \rightarrow \partial \mho_{s}^{n}\left(\right.$ since, for every $i=1, \ldots, h,\left.\bar{\varphi}^{-1} \circ \psi \circ \varphi\right|_{\partial \mathbb{D}_{(i)}^{n-1}}$ extends to the whole $(n-1)-$ ball $\mathbb{D}_{(i)}^{n-1}$ of $\left.\partial \mho_{s}^{n}\right)$; then, $T$ may be extended to a self-homeomorphism $\tilde{T}: \mathbb{Y}_{s}^{n} \rightarrow \mathbb{Y}_{s}^{n}$.

Finally, the proof follows by considering the homeomorphism

$$
\tilde{\psi} \cup \tilde{T}: M^{n}=\mathbb{Y}_{r}^{n} \cup_{\varphi} \mathbb{Y}_{s}^{n} \longrightarrow \mathbb{Y}_{r}^{n} \cup_{\bar{\varphi}} \mathbb{Y}_{s}^{n}=\#_{s}\left(\mathbb{S}^{n-1} \times \mathbb{S}^{1}\right) \#^{(h)} \mathbb{U}_{r-s}^{n} .
$$

3. Technical results about crystallizations and regular genus. In order to understand how to compute the combinatorial invariant "regular genus", we have to briefly recall the basic notions of the representation theory of PL $n$-manifolds (for every $n$ ) by edge-coloured graphs; for a detailed treatment, see [FGG], [Co], [LM] or [V].

According to [FGG], we say that a finite ball-complex $K$ is a pseudocomplex iff:

- $|K|=\amalg\{\operatorname{int}(B) / B \in K\}$ (where $\amalg$ denotes a disjoint union);

- if $A, B \in K$, then $A \cap B$ is a union of balls of $K$;

- for each $m$-ball $A \in K$, the poset $\{B \in K / B \subseteq A\}$, ordered by inclusion, is isomorphic with the lattice of all faces of the standard $m$-simplex.

Note that, in general, a pseudocomplex is not a simplicial complex, since its elements may intersect in more than one face; notwithstanding this, we will always refer to an $m$-ball $B \in K$ as to an $m$-simplex of $K$. Further, note that the (well defined) barycentric subdivision of any pseudocomplex is actually a simplicial complex.

Now, it is easy to check that every PL $n$-manifold $M^{n}$ admits a coloured pseudodissection $(K, \xi)$, i.e. a pseudocomplex $K$ with $|K| \cong M^{n}$, endowed with a vertex labelling $\xi$ by colours of the set $\Delta_{n}=\{0,1, \ldots, n\}$, which is injective on every $n$-simplex of $K .^{4}$ Moreover, a coloured pseudodissection $(K, \xi)$ of $M^{n}$ may be combinatorially visualized by means of an $(n+1)$-coloured graph $(\Gamma, \gamma)$, where the multigraph $\Gamma=(V(\Gamma), E(\Gamma))$ is the 1-skeleton of the (well defined) ball-complex dual to $K$, and the edge-coloration $\gamma: E(\Gamma) \rightarrow \Delta_{n}$ is given by $\gamma(e)=c$ if the edge $e \in E(\Gamma)$ is dual to an $(n-1)$-simplex of $K$ having no vertex labelled by colour $c ;(\Gamma, \gamma)$ is said to represent $M^{n}$, since the reversed process allows to recontruct the associated coloured pseudodissection $(K, \xi)$-and hence $M^{n}$-from it.

Note that, for every subset $\mathcal{B} \subset \Delta_{n}$, with $\# \mathcal{B}=m$, a (possibly disconnected) $m$ coloured graph $\Gamma_{\mathcal{B}}=\left(V(\Gamma), \gamma^{-1}(\mathcal{B})\right)$ is well defined; by construction, its connected components (which are said to be $\mathcal{B}$-residues of $\Gamma$ ) are in bijection with the $(n-m)$ simplices of $K$ whose vertices are labelled by $\Delta_{n}-\{\mathcal{B}\}$. The number of $\mathcal{B}$-residues of $\Gamma$ is usually denoted by $g_{\mathcal{B}}$; in particular, if $\mathcal{B}=\{i, j\}$ (resp. $\mathcal{B}=\{i, j, k\}$ ) (resp. $\mathcal{B}=\Delta_{n}-\{i\}$ ), we shall often write $g_{i j}$ (resp. $g_{i j k}$ ) (resp. $\left.g_{\hat{i}}\right)$ instead of $g_{\mathcal{B}}$, and $g_{\hat{i} j}$ (resp. $\left.g_{\hat{i} \hat{k}}\right)\left(\right.$ resp. $\left.g_{i}\right)$ instead of $g_{\Delta_{n}}-\mathcal{B}$.

Let $\mathcal{G}_{n+1}$ be the class of $(n+1)$-coloured graphs $(\Gamma, \gamma)$ which are regular with respect to the "last" colour n, i.e. so that $\Gamma_{\hat{n}}$ is a regular graph of degree $n$.

A theorem of [P], together with its subsequent improvements (see [FGG] and its bibliography), ensures that every PL $n$-manifold $M^{n}$ may be represented by an $(n+1)$-coloured

\footnotetext{
${ }^{4}$ For example, $K$ may be the barycentric subdivision of any simplicial triangulation $H$ of $M^{n}$, and $\xi$ may associate to every vertex $v$ of $K$ the dimension of the simplex of $H$ whose barycenter is $v$.
} 
graph $(\Gamma, \gamma) \in \mathcal{G}_{n+1}$. More precisely, if the boundary $\partial M^{n}$ is either empty or connected, $M^{n}$ is proved to admit a contracted triangulation (resp. a crystallization), i.e. a coloured pseudodissection $(K, \xi)$ containing exactly $n+1$ vertices (resp. i.e. an $(n+1)$-coloured $\operatorname{graph}(\Gamma, \gamma) \in \mathcal{G}_{n+1}$ with $g_{\hat{i}}=1$, for every $\left.i \in \Delta_{n}\right)$; on the other hand, if $\partial M^{n}$ has $h \geq 2$ connected components, then any contracted triangulation of $M^{n}$ contains exactly $n h+1$ vertices, and any crystallization of $M^{n}$ satisfies $g_{\hat{n}}=1$ and $g_{\hat{i}}=1$ for every $i \in \Delta_{n-1}$.

Obviously, if $(\Gamma, \gamma) \in \mathcal{G}_{n+1}$ has order $2 p=\# V(\Gamma)$, then $(\Gamma, \gamma)$ contains $2 \dot{p} \leq 2 p$ degree $n+1$ vertices (which are said to be internal vertices) and $2 \bar{p}=2 p-2 \dot{p} \geq 0$ degree $n$ vertices (which are said to be boundary vertices); further, if $\mathcal{B} \subset \Delta_{n}$, $(\Gamma, \gamma)$ contains $\dot{g}_{\mathcal{B}} \leq g_{\mathcal{B}}$ internal $\mathcal{B}$-residues (i.e. $\mathcal{B}$-residues which result to be regular graphs), while the other $\bar{g}_{\mathcal{B}}=g_{\mathcal{B}}-\dot{g}_{\mathcal{B}} \geq 0$ ones are said to be boundary $\mathcal{B}$-residues. ${ }^{5}$

It is not difficult to check that, if $(\Gamma, \gamma) \in \mathcal{G}_{n+1}$ represents a PL $n$-manifold $M^{n}$, then every internal (resp. boundary) $\mathcal{B}$-residue, with $\# \mathcal{B}=m \leq n$, belongs to $\mathcal{G}_{m}$ and represents the $(m-1)$-sphere $\mathbb{S}^{m-1}$ (resp. the $(m-1)$-ball $\left.\mathbb{D}^{m-1}\right)$; moreover, $M^{n}$ is orientable if and only if $(\Gamma, \gamma)$ is bipartite, and $M^{n}$ is closed if and only if $(\Gamma, \gamma)$ has no boundary vertex.

On the other hand, if $(\Gamma, \gamma) \in \mathcal{G}_{n+1}$ represents a PL $n$-manifold $M^{n}$ with (non-empty) boundary $\partial M^{n}$, then $\partial M^{n}$ is represented by the so called boundary $\operatorname{graph}^{6}\left(\partial \Gamma,{ }^{2} \gamma\right) \in \mathcal{G}_{n}$, whose vertices are in bijection with the boundary vertices of $\Gamma$, and whose $i$-coloured edges $\left(i \in \Delta_{n-1}\right)$ are in bijection with the boundary $\{i, n\}$-residues of $\Gamma$; thus, for every $\mathcal{B} \subset \Delta_{n-1}$, the number ${ }^{g_{\mathcal{B}}}$ of $\mathcal{B}$-residues of $\partial \Gamma$ equals the number $\bar{g}_{\mathcal{B} \cup\{n\}}$ of boundary $(\mathcal{B} \cup\{n\})$-residues of $\Gamma$.

In $\left[\mathrm{G}_{1}\right]$ and $\left[\mathrm{G}_{2}\right]$ it is proved that every bipartite $(n+1)$-coloured graph $(\Gamma, \gamma) \in \mathcal{G}_{n+1}$ "regularly embeds" (see $\left[\mathrm{G}_{1}\right]$ for details) onto an orientable surface $F_{\varepsilon}$, which depends on the choice of a cyclic permutation $\varepsilon=\left(\varepsilon_{1}, \varepsilon_{2}, \ldots \varepsilon_{n-1}, \varepsilon_{n}=n\right)$ of the colour set $\Delta_{n}$; the regular genus $\varrho_{\varepsilon}(\Gamma)$ of $(\Gamma, \gamma)$ with respect to $\varepsilon$ is defined as the genus of $F_{\varepsilon}$, and is computable-also in the generalized case of a disconnected graph with $g \geq 2$ components - by means of the following combinatorial formula:

$$
\sum_{i \in \mathbb{Z}_{n}} \dot{g}_{\varepsilon_{i} \varepsilon_{i+1}}+(1-n) \frac{\dot{p}}{2}+(2-n) \frac{\bar{p}}{2}+{ }^{\partial} g_{\varepsilon_{0} \varepsilon_{n-1}}=2 g-2 \varrho_{\varepsilon}(\Gamma) .
$$

Finally, the regular genus $\mathcal{G}\left(M^{n}\right)$ of an orientable PL $n$-manifold $M^{n}$ is defined to be the minimum value of $\varrho_{\varepsilon}(\Gamma)$, for every crystallization $(\Gamma, \gamma)$ of $M^{n}$ and for every cyclic permutation $\varepsilon$ of $\Delta_{n}$.

If $(\Gamma, \gamma) \in \mathcal{G}_{n+1}$ represents an orientable PL $n$-manifold $M^{n}$, and $\varepsilon=\left(\varepsilon_{1}, \varepsilon_{2}, \ldots, \varepsilon_{n-1}\right.$, $\left.\varepsilon_{n}=n\right)$ is a fixed cyclic permutation of $\Delta_{n}$, then every subset $\mathcal{B} \subset \Delta_{n}$ inherits an induced cyclic permutation $\varepsilon_{\mathcal{B}}$; thus, formula (1) may be applied also to compute the regular genus $\varrho_{\varepsilon_{\mathcal{B}}}\left(\Gamma_{\mathcal{B}}\right)$ for every (possibly disconnected) subgraph $\Gamma_{\mathcal{B}}$, and the regular genus $\varrho_{\varepsilon_{\hat{n}}}(\partial \Gamma)$ for the (possibly disconnected) boundary graph $\left(\partial \Gamma,{ }_{\gamma}\right)$.

\footnotetext{
5 Note that, if $n \notin \mathcal{B}$, every $\mathcal{B}$-residue is an internal residue.

6 Obviously, the boundary graph is a possibly disconnected graph, having as many components as the boundary manifold $\partial M^{n}$.
} 
From now on-for sake of notational simplicity-the fixed cyclic permutation $\varepsilon=$ $\left(\varepsilon_{1}, \varepsilon_{2}, \ldots \varepsilon_{n-1}, \varepsilon_{n}=n\right)$ will be assumed to be the identity on $\Delta_{n}$; thus, we shall always write $i$ instead of $\varepsilon_{i}$, for every $i \in \Delta_{n}$, and $\varrho$ (resp. $\varrho_{\mathcal{B}}$ ) (resp. ${ }^{\partial} \varrho$ ) instead of $\varrho_{\varepsilon}(\Gamma)$ (resp. $\left.\varrho_{\varepsilon_{\mathcal{B}}}\left(\Gamma_{\mathcal{B}}\right)\right)\left(\right.$ resp. $\left.\varrho_{\varepsilon_{\tilde{n}}}(\partial \Gamma)\right)$.

For our purposes, some general formulae — which extend similar ones already proved in $[\mathrm{CG}]$ and $\left[\mathrm{C}_{2}\right]$ —result to be useful.

PROPOSITION 3. With the above notations, the following relations hold:

(2) $g_{c-1, c+1}=g_{c-1, c, c+1}+\left(\varrho-\varrho_{\hat{c}}\right)+\left(g_{\hat{c}}-g\right) \quad \forall c \notin\{0, n-1\}$;

(20) $\dot{g}_{n, 1}=\dot{g}_{n, 0,1}+\left(\varrho-\varrho_{\hat{0}}\right)+\left(g_{\hat{0}}-g\right)-\partial_{g_{n-1,1}}+\partial_{g_{n-1,0,1}}$;

$\left(2_{n-1}\right) \dot{g}_{n-2, n}=\dot{g}_{n-2, n-1,0}+\left(\varrho-\varrho_{\hat{n}-1}\right)+\left(g_{\hat{n}-1}-g\right)-{ }_{g_{0, n-2}}+\partial_{g_{n-2, n-1,0}}$;

(3) $\dot{g}_{i-1, i+1}=\dot{g}_{i-1 i, i+1}+\left(\varrho-\varrho_{\hat{i}}\right)-\left({ }^{\partial} \varrho-{ }^{\partial} \varrho_{\hat{i}}\right)-\left(g-\partial_{g}-\dot{g}_{\hat{i}}\right)$ for $i \in\{0, n-1\}$;

(4) $g_{\hat{i} j}=\left(g_{\hat{i}}+g_{\hat{j}}-g\right)+\varrho-\varrho_{\hat{i}}-\varrho_{\hat{j}}+\varrho_{\hat{i} j}$ for every $i, j$ non-consecutive in $\Delta_{n}$, with $\{i, j\} \neq\{0, n-1\}$

(5) $\dot{g}_{\hat{i} \hat{j}}=\left[\dot{g}_{\hat{i}}+\dot{g}_{\hat{j}}-\left(g-{ }_{g} g\right)\right]+\left(\varrho-{ }^{\partial} \varrho\right)-\left(\varrho_{\hat{i}}-{ }^{\partial} \varrho_{\hat{i}}\right)-\left(\varrho_{\hat{j}}-{ }^{\partial} \varrho_{\hat{j}}\right)+\left(\varrho_{\hat{i}}-{ }^{\partial} \varrho_{\hat{i}}\right)$ for every $i, j$ non-consecutive in $\Delta_{n-1}$.

PROOF. First of all, note that the Euler characteristic computation of all pseudocomplexes represented by $\{i, j, k\}$-residues of $\Gamma$ easily yields the following relations:

(6) $2 g_{i, j, k}=g_{i, j}+g_{i, k}+g_{j, k}-\frac{p}{2} \quad \forall i, j, k \in \Delta_{n-1}$;

(6') $2 \dot{g}_{i, j, n}+\partial_{g_{i, j}}=g_{i, j}+\dot{g}_{i, n}+\dot{g}_{j, n}-\frac{\dot{p}}{2} \quad \forall i, j \in \Delta_{n-1}$.

Then formula ( $2_{c}$ ), for every $c \in \Delta_{n}$, may be obtained by applying relation (1) both to $(\Gamma, \gamma) \in \mathcal{G}_{n+1}$ and to its subgraph $\Gamma_{\hat{c}}$, and by making suitable use of formulae (6) and/or $\left(6^{\prime}\right)$.

Further, note that, for $i \in\{0, n-1\}$, the comparison between formula $\left(2_{i}\right)$ applied to an $(n+1)$-coloured graph and the same formula applied to its boundary graph, directly yields formula $\left(3_{i}\right)$.

As far as relation (4) is concerned, it is sufficient to apply formula $\left(2_{i}\right)$ both to $(\Gamma, \gamma)$ and to its subgraph $\Gamma_{\hat{j}}$; finally, formula (5) easily follows from relation (4), applied both to $(\Gamma, \gamma)$ and to its boundary graph.

Let us now restrict our attention to the case of $(\Gamma, \gamma) \in \mathcal{G}_{6}$ being a crystallization of an orientable PL 5-manifold $M^{5}$, with $h \geq 1$ boundary components; when we apply the above formulae (2), (3), (4), (5) to $(\Gamma, \gamma)$ or to the subgraph $\Gamma_{\hat{k}}\left(k \in \Delta_{5}\right)$, we have to remind that $g=g_{\hat{\zeta}}=\dot{g}_{\hat{\zeta}}=1$, while $\partial_{g}=g_{\hat{i}}=h$ and $\dot{g}_{\hat{i}}=0$ for every $i \in \Delta_{4}$, and that $\varrho_{\hat{i} \hat{j} \hat{k}}={ }^{\partial} \varrho_{\hat{i} \hat{j}}=0$ (since every 3 -coloured graph representing either $\mathbb{S}^{2}$ or $\mathbb{D}^{2}$ has always regular genus zero). Moreover, the following statement lists other interesting relations.

PROPOSITION 4. Let $(\Gamma, \gamma) \in \mathcal{G}_{6}$ be a crystallization of an orientable PL 5-manifold $M^{5}$, with connected boundary $\partial M^{5} \neq \emptyset$. For every cyclic permutation $\varepsilon=\left(\varepsilon_{0}, \ldots, \varepsilon_{4}\right.$, $\left.\varepsilon_{5}=5\right)$ of $\Delta_{5}$, we have:

(7) $2 \varrho_{\hat{i}}={ }^{\partial} \varrho_{\hat{i}}+\sum_{j \neq i} \varrho_{i j}$ for every $i \in \Delta_{4}$;

(8) $2 \varrho_{\hat{S}}=\sum_{j \neq 5} \varrho_{\hat{S} \hat{j}}$;

(9) $\dot{g}_{\hat{0} \hat{2} \hat{4}}=2\left(\varrho-{ }^{\partial} \varrho\right)-\left(\varrho_{\hat{0}}-{ }^{\partial} \varrho_{\hat{0}}\right)-\left(\varrho_{\hat{2}}-{ }^{\partial} \varrho_{\hat{2}}\right)-\left(\varrho_{\hat{4}}-{ }^{\partial} \varrho_{\hat{4}}\right)+2 h-2 \geq 2 h-2$; 
(10) $g_{\hat{1} \hat{3} \hat{j}}=2 \varrho-\left(\varrho_{\hat{1}}+\varrho_{\hat{3}}+\varrho_{\hat{\zeta}}\right)+2 h-1 \geq 2 h-1$.

PROOF. In $\left[\mathrm{C}_{2} ;\right.$ Lemma 8], the Euler characteristic of a (possibly disconnected, with $\dot{g}$ closed components and $\bar{g}$ bounded components) 4-manifold $M^{4}$ has been computed by means of the regular genera associated to any 5-coloured graph representing $M^{4}$ : $X\left(M^{4}\right)=2 \dot{g}+\bar{g}-2 \varrho+{ }^{\partial} \varrho+\sum_{i} \varrho_{\hat{i}}$.

Hence, formulae (7) and (8) directly follow, since-in the hypothesis of the statement- $\Gamma_{\hat{i}}$, with $i \in \Delta_{4}$, consists of $h \geq 1$ connected components, each one representing $\mathbb{D}^{4}$, while $\Gamma_{\hat{s}}$ is a connected graph representing $\mathbb{S}^{4}$.

Let us now consider the 6-coloured graph $(\Gamma, \gamma) \in \mathcal{G}_{6}$ of the statement; summing up relations $\left(2_{2}\right),\left(3_{0}\right)$ and $\left(3_{4}\right)$, together with an appropriate use of formulae $(6)$ and $\left(6^{\prime}\right)$, gives:

$$
\begin{aligned}
g_{13}+\dot{g}_{51}+ & \dot{g}_{35} \\
= & \dot{g}_{501}+\dot{g}_{345}+g_{123}+3 \varrho-2^{\partial} \varrho-\varrho_{\hat{2}}-\left(\varrho_{\hat{0}}-{ }^{\partial} \varrho_{\hat{0}}\right)-\left(\varrho_{\hat{4}}-{ }^{\partial} \varrho_{\hat{4}}\right)+3 h-3 \\
= & \frac{1}{2}\left(g_{01}+\dot{g}_{05}+\dot{g}_{15}+g_{12}+g_{13}+g_{23}+g_{34}+\dot{g}_{35}+\dot{g}_{45}-{ }_{g_{01}}-{ }_{g_{34}}\right) \\
& \quad-\frac{3}{4} \dot{p}-\frac{1}{4} \bar{p}+3 \varrho-2{ }^{\partial} \varrho-\varrho_{\hat{2}}-\left(\varrho_{\hat{0}}-{ }^{\partial} \varrho_{\hat{0}}\right)-\left(\varrho_{\hat{4}}-{ }^{\partial} \varrho_{\hat{4}}\right)+3 h-3 .
\end{aligned}
$$

Now, formula (1) yields:

$$
\begin{aligned}
\frac{1}{2}\left(g_{13}+\dot{g}_{51}+\dot{g}_{35}=(\right. & \left.-\varrho-\frac{1}{2} \partial g_{04}+\dot{p}+\frac{3}{4} \bar{p}\right)-\frac{1}{2}\left({ }^{\partial} g_{01}+{ }^{\partial} g_{34}\right) \\
& -\frac{3}{4} \dot{p}-\frac{1}{4} \bar{p}+3 \varrho-2{ }^{\partial} \varrho-\varrho_{\hat{2}}-\left(\varrho_{\hat{0}}-{ }^{\partial} \varrho_{\hat{0}}\right) \\
& -\left(\varrho_{\hat{4}}-{ }^{\partial} \varrho_{\hat{4}}\right)+3 h-3 .
\end{aligned}
$$

Thus, the computation of $\dot{g}_{\hat{0} \hat{2} \hat{4}}$ given in (9) follows from a further use of relation ( $\left.6^{\prime}\right)$, together with an application of formula (1) to the 4-coloured graph $\partial \Gamma_{\hat{2}}$ :

$$
\begin{aligned}
\dot{g}_{\hat{0} \hat{2} \hat{4}}= & \dot{g}_{135} \\
= & 1+2\left(\varrho-{ }^{\partial} \varrho\right)-\varrho_{\hat{2}}-\left(\varrho_{\hat{0}}-{ }^{\partial} \varrho_{\hat{0}}\right)-\left(\varrho_{\hat{4}}-{ }^{\partial} \varrho_{\hat{4}}\right) \\
& +\frac{1}{2} \bar{p}-\frac{1}{2}\left({ }^{\partial} g_{01}+{ }^{\partial} g_{34}+{ }^{\partial} g_{13}+{ }^{\partial} g_{04}\right)+3 h-3 \\
= & 2\left(\varrho-{ }^{\partial} \varrho\right)-\left(\varrho_{\hat{0}}-{ }^{\partial} \varrho_{\hat{0}}\right)-\left(\varrho_{\hat{2}}-{ }^{\partial} \varrho_{\hat{2}}\right)-\left(\varrho_{\hat{4}}-{ }^{\partial} \varrho_{\hat{4}}\right)+2 h-2 .
\end{aligned}
$$

Further, it is not difficult to get the inequality $\dot{g}_{\hat{0} \hat{\imath} \hat{4}} \geq 2 h-2$, by recalling that the pseudocomplex $K$ (which has $h \geq 1$ boundary components) is strongly connected: for example, in case $h=2$, two internal triangles $\sigma$ and $\tau$ surely exist, so that $\sigma$ (resp. $\tau$ ) has its $i$-labelled and $j$-labelled vertices (resp. its $i$-labelled vertex) on one boundary component and its $k$-labelled vertex (resp. its $j$-labelled and $k$-labelled vertices) on the other boundary component, $\{i, j, k\}$ being a suitable permutation of $\{0,2,4\}$. 
On the other hand, summing up relations $\left(2_{1}\right),\left(2_{3}\right)$ and $\left(2_{5}\right)$, together with an appropriate use of of formulae (6) and $\left(6^{\prime}\right)$, gives:

$$
\begin{aligned}
g_{02}+g_{24}+g_{40}= & g_{012}+g_{234}+g_{450}+3 \varrho-\left(\varrho_{\hat{1}}+\varrho_{\hat{3}}+\varrho_{\hat{\zeta}}\right)+2 h-2 \\
= & \frac{1}{2}\left(g_{01}+g_{12}+g_{02}+g_{23}+g_{34}+g_{24}+\dot{g}_{45}+\dot{g}_{50}+g_{40}-\partial_{g_{04}}\right) \\
& \quad-\frac{3}{4} \dot{p}-\frac{1}{2} \bar{p}+3 \varrho-\left(\varrho_{\hat{1}}+\varrho_{\hat{3}}+\varrho_{\hat{5}}\right)+2 h-2 .
\end{aligned}
$$

Now, formula (1) yields:

$$
\begin{aligned}
\frac{1}{2}\left(g_{02}+g_{24}+g_{40}\right)=(1 & \left.-\varrho-\frac{1}{2} \partial g_{04}+\dot{p}+\frac{3}{4} \bar{p}\right) \\
& +\frac{1}{2} \partial_{g_{04}}-\frac{3}{4} \dot{p}-\frac{1}{2} \bar{p}+3 \varrho-\left(\varrho_{\hat{1}}+\varrho_{\hat{\jmath}}+\varrho_{\hat{\zeta}}\right)+2 h-2 .
\end{aligned}
$$

Hence, the computation of $g_{\hat{1}\{\hat{s}}$ given in (10) may be completed by a further use of relation (6).

Finally, the inequality $g_{\hat{1} \hat{3} \mathfrak{5}} \geq 2 h-1$ is a consequence of the existence of $h \geq 1$ boundary components (each one containing at least an edge between its 1-labelled and 3-labelled vertices) and of the strong connectedness of the pseudocomplex $K$ (which implies the existence of at least $h-1$ edges, with 1-labelled and 3-labelled end-points, "connecting" the $h$ boundary components.

If $(\Gamma, \gamma) \in \mathcal{G}_{6}$ is assumed to be a crystallization of a PL 5-manifold $M^{5}$ (with $h \geq 1$ boundary components), then the vertex set of the associated coloured pseudodissection $(K, \xi)$ is

$$
V(K)=\left\{v_{0}^{(1)}, \ldots, v_{0}^{(h)}, v_{1}^{(1)}, \ldots, v_{1}^{(h)}, v_{2}^{(1)}, \ldots, v_{2}^{(h)}, v_{3}^{(1)}, \ldots, v_{3}^{(h)}, v_{4}^{(1)}, \ldots, v_{4}^{(h)}, v_{5}\right\},
$$

with $\xi\left(v_{i}\right)=i$, for every $i \in \Delta_{5}$.

For every $\{i, j\} \subset \Delta_{5}$ (resp. $\{i, j, k\} \subset \Delta_{5}$ ), let $K(i, j)$ (resp. $\left.K(i, j, k)\right)$ denote the 1dimensional (resp. 2-dimensional) subcomplex of $K$ generated by $\{v \in V(K) / \xi(v) \in$ $\{i, j\}\}$ (resp. $\{v \in V(K) / \xi(v) \in\{i, j, k\}\}$ ), and let $N(i, j)$ (resp. $N(i, j, k))$ be a regular neighbourhood of $|K(i, j)|$ (resp. $|K(i, j, k)|)$ into the polyhedron $|K|$. Moreover, if $\{i, j\} \subset$ $\Delta_{4}$ (resp. $\{i, j, k\} \subset \Delta_{4}$ ), let $\dot{K}(i, j)$ (resp. $\left.\dot{K}(i, j, k)\right)$ denote the subcomplex of $K(i, j)$ (resp. $K(i, j, k))$ consisting only of the 1-simplices (resp. 2-simplices) which are internal in $K$, together with their faces, and $\operatorname{set}^{\partial} K(i, j)=K(i, j) \cap \partial K\left(\right.$ resp. $\left.{ }^{\partial} K(i, j, k)=K(i, j, k) \cap \partial K\right)$.

Note that, if $\left\{\{i, j, k\},\left\{i^{\prime}, j^{\prime}, k^{\prime}\right\}\right\}$ is a partition of $\Delta_{5}$, then the 5-dimensional submanifolds $N(i, j, k)$ and $N\left(i^{\prime}, j^{\prime}, k^{\prime}\right)$ give rise to a decomposition $M^{5}=N(i, j, k) \cup_{\varphi} N\left(i^{\prime}, j^{\prime}, k^{\prime}\right), \varphi$ being a (partial) boundary identification. Thus, it becomes interesting to get information about the topological structure of the above described subcomplexes of $K$.

LEMMA 5. Let $(\Gamma, \gamma) \in \mathcal{G}_{6}$ be a crystallization of an orientable PL 5-manifold $M^{5}$, with $h \geq 1$ boundary components.

a) If there exist $i, j, k \in \Delta_{5}$, with $i, j$ non-consecutive in $\Delta_{5}-\{k\}$ and $\{i, j\} \neq\{0,4\}$, such that $\varrho_{\hat{k}}-\varrho_{\hat{i} \hat{k}}-\varrho_{\hat{j} \hat{k}}=0$, then $K(i, j, k)$ collapses to $K(i, j)$; 
b) if there exist $i, j, k \in \Delta_{4}$, with $i, j$ non-consecutive in $\Delta_{4}-\{k\}$, such that $\varrho_{\hat{k}}-{ }^{\partial} \varrho_{\hat{k}}-$ $\varrho_{\hat{i} \hat{k}}-\varrho_{\hat{j} \hat{k}}=0$, then $K(i, j, k)$ collapses to ${ }^{\partial} K(i, j, k) \cup \dot{K}(i, j)$.

PROOF. Formula (4), applied to the residue $\Gamma_{\hat{k}}$ of $(\Gamma, \gamma)$, gives $g_{\hat{i} \hat{j} \hat{k}}=\left(g_{\hat{i} \hat{k}}+g_{\hat{j} \hat{k}}-g_{\hat{k}}\right)+$ $\left(\varrho_{\hat{k}}-\varrho_{\hat{i} \hat{k}}-\varrho_{\hat{k} \hat{k}}\right)$, for every $i, j$ non-consecutive in $\Delta_{5}-\{k\}$, with $\{i, j\} \neq\{0,4\}$. Thus, if $\varrho_{\hat{k}}-\varrho_{\hat{i} \hat{k}}-\varrho_{\hat{j} \hat{k}}=0$ is assumed, $g_{\hat{i} \hat{k}}=g_{\hat{i} \hat{k}}+g_{\hat{j} \hat{k}}-g_{\hat{k}}$ obviously holds, and $K(i, j, k)$ surely collapses to $K(i, j)$ by a trivial extension of [ $\mathrm{C}_{1}$; Lemma 5] (or [CG; Lemma 1a]).

On the other hand, formula (5), applied to the residue $\Gamma_{\hat{k}}$ of $(\Gamma, \gamma)$ (with $\left.k \neq 5\right)$, gives $\dot{g}_{\hat{i} \hat{j} \hat{k}}=\left(\dot{g}_{\hat{i} \hat{k}}+\dot{g}_{\hat{j} \hat{k}}\right)+\left(\varrho_{\hat{k}}-{ }^{\partial} \varrho_{\hat{k}}-\varrho_{\hat{i} \hat{k}}-\varrho_{\hat{j} \hat{k}}\right)$, for every $i, j$ non-consecutive in $\Delta_{4}-\{k\}$. Thus, if $\varrho_{\hat{k}}-\varrho_{\varrho_{\hat{k}}}-\varrho_{\hat{i} \hat{k}}-\varrho_{\hat{j} \hat{k}}=0$ is assumed, $\dot{g}_{\hat{i} \hat{j} \hat{k}}=\dot{g}_{\hat{i} \hat{k}}+\dot{g}_{\hat{j} \hat{k}}$ obviously holds.

Note that, since the edges of $K(i, j)$ are a set of generators for the fundamental group (see [CP]), every edge of $\dot{K}(i, k)$ and $\dot{K}(j, k)$ is face of a triangle of $\dot{K}(i, j, k)$, at least; further, the fact that $\Gamma_{\hat{k}}$ has no internal component implies the existence of at least a triangle in $\dot{K}(i, j, k)$ having its $(s, k)$-face, for $s \in\{i, j\}$, on the boundary of $K$.

It is now easy to check that, if $\dot{g}_{\hat{s} \hat{k}} \geq \dot{g}_{\hat{r} \hat{k}}$, with $\{r, s\}=\{i, j\}$, then the edges of $\dot{K}(s, k)$ are faces of at most $\dot{g}_{\hat{i} \hat{k} k}-1=\dot{g}_{\hat{i} \hat{k}}+\dot{g}_{\hat{j} \hat{k}}-1 \leq 2 \dot{g}_{\hat{s} \hat{k}}-1$ triangles; thus, at least $\dot{g}_{\hat{s} \hat{k}}-\dot{g}_{\hat{r} \hat{k}}+1$ triangles of $\dot{K}(i, j, k)$ may collapse from their "free" internal $(s, k)$-face. On the other hand, if $\dot{g}_{\hat{s} \hat{k}}<\dot{g}_{\hat{r} \hat{k}}$, some triangle of $\dot{K}(i, j, k)$ may obviously collapse from its "free" internal $(r, k)$-face. Moreover, since the above considered collapses do not affect the property $\dot{g}_{\hat{k}}=0$ and leave $K(i, j)$ fixed, the process may be iterated, until each triangle of $\dot{K}(i, j, k)$ collapses either from its "free" internal $(i, k)$-face, or from its "free" internal $(j, k)$-face. Finally, since relation $\dot{g}_{\hat{i} \hat{j} \hat{k}}=\dot{g}_{\hat{i} \hat{k}}+\dot{g}_{\hat{\hat{k}} \hat{k}}$ holds at every step, the result of the whole collapsing process is nothing but ${ }^{\partial} K(i, j, k) \cup \dot{K}(i, j)$, as statement (b) claims.

The following Lemma makes use of Lemma 5 to prove that, under suitable combinatorial hypothesis, the decomposition $M^{5}=N(i, j, k) \cup_{\varphi} N\left(i^{\prime}, j^{\prime}, k^{\prime}\right)$ induced by a partition $\left\{\{i, j, k\},\left\{i^{\prime}, j^{\prime}, k^{\prime}\right\}\right\}$ of $\Delta_{5}$, is indeed a $(1,4)$-handle presentation.

LEMMA 6. Let $(\Gamma, \gamma) \in \mathcal{G}_{6}$ be a crystallization of an orientable PL 5-manifold $M^{5}$, with $h \geq 1$ boundary components. If there exist $i, j \in \Delta_{5}$ such that $\varrho_{i \hat{j}}=0$, then $M^{5} \cong$ $\#_{a}\left(\mathbb{S}^{4} \times \mathbb{S}^{1}\right) \#^{(h)} \mathbb{H}_{b}$, for suitable $a, b$, with $0 \leq b \leq{ }^{\partial}$, and $0 \leq a \leq \varrho-{ }^{\partial} \varrho$.

PROOF. Let us assume the existence of $i, j \in \Delta_{5}$, with $i \neq 5$, such that $\varrho_{\hat{i} j}=0$, and let $\left(0^{(i j)}, 1^{(i j)}, 2^{(i j)}, 3^{(i j)}\right)$ denote the (ordered) 4 -tuples induced by $\varepsilon$ on $\Delta_{5}-\{i, j\}$; then, formulae (7) and/or (8) imply

$$
\begin{aligned}
& \left(\varrho_{\hat{i}}-\varrho_{\hat{i}}-\varrho_{\hat{0}^{(i j)}}-\varrho_{\hat{2}^{(i)} \hat{i}}\right)+\left(\varrho_{\hat{i}}-\varrho_{\hat{1}(i) \hat{i}}-\varrho_{\hat{\zeta}^{(i j) \hat{i}}}\right)=\varrho_{\hat{i} \hat{j}}=0 \\
& \left(\varrho_{\hat{j}}-{ }^{\partial} \varrho_{\hat{j}}-\varrho_{0^{(i j)} \hat{j}}-\varrho_{\hat{2}^{(i j)}} \hat{j}\right)+\left(\varrho_{\hat{j}}-\varrho_{\hat{1}^{(i j)}}-\varrho_{\hat{\zeta}^{(i j} \hat{j}}\right)=\varrho_{\hat{i} \hat{j}}=0 \text {, }
\end{aligned}
$$

where-for sake of conciseness-we assume ${ }^{\partial} \varrho_{\hat{j}}=0$ in case $j=5$.

Since the four addenda are non-negative integer (see relations (4) and (5), together with the trivial inequalities $g_{\hat{i} j} \geq g_{\hat{i}}+g_{\hat{j}}-g$ and $\dot{g}_{\hat{i} \hat{j}} \geq \dot{g}_{\hat{i}}+\dot{g}_{\hat{j}}-\left(g-\partial_{g}\right)$ ), we have both $\varrho_{\hat{j}}-\varrho_{\hat{1}(i) \hat{j}}-\varrho_{\hat{\zeta}(i) \hat{j}}=0$ and $\varrho_{\hat{i}}-{ }^{\partial} \varrho_{\hat{i}}-\varrho_{\hat{0}^{(i j) \hat{i}}}-\varrho_{\hat{2}^{(i j) \hat{i}}}=0$; thus, Lemma 5 implies that both $K\left(j, 1^{(i j)}, 3^{(i j)}\right)$ collapses to $K\left(1^{(i j)}, 3^{(i j)}\right)$ and $K\left(i, 0^{(i j)}, 2^{(i j)}\right)$ collapses to 
$\dot{K}\left(0^{(i j)}, 2^{(i j)}\right) \cup{ }^{\partial} K\left(i, 0^{(i j)}, 2^{(i j)}\right)$. Moreover, since the regular neighbourhood of any simplex of ${ }^{\partial} K\left(i, 0^{(i j)}, 2^{(i j)}\right)$ is a collar of a 4-ball embedded in $\partial M^{5}$, the decomposition of $M^{5}$ induced by the partition $\left\{\left\{j, 1^{(i j)}, 3^{(i j)}\right\},\left\{i, 0^{(i j)}, 2^{(i j)}\right\}\right\}$ of $\Delta_{5}$ may be thought of as $M^{5}=\mathbb{Y}_{r} \cup_{\varphi} \mathbb{Y}_{s}$, for a suitable $\varphi: \partial \mathbb{Y}_{s}-\bigcup_{i=1}^{h} \operatorname{int}\left(\mathbb{D}_{(i)}^{4}\right) \rightarrow \partial \mathbb{Y}_{r}$, with $r=g_{\left.\hat{1}^{(j)},\right\}^{(i)}}-$ $\left(g_{\hat{1}^{(i)}}+g_{\hat{\xi}(i j)}-1\right)$ (recall that a maximal tree of $K\left(1^{(i j)}, 3^{(i j)}\right)$ is uninfluent) and $s=\dot{g}_{\hat{0}^{(i)}, 2^{(i j)}}-$ $\left(g_{0_{(i)}}+g_{\hat{2}_{(i j)}}-1\right)+h\left(\right.$ recall that a maximal tree of $K\left(0^{(i j)}, 2^{(i j)}\right)$ is uninfluent, too, and that ${ }^{\partial} K\left(i, 0^{(i j)}, 2^{(i j)}\right)$ has $h$ connected components).

Now, $M^{5} \cong \#_{a}\left(\mathbb{S}^{4} \times \mathbb{S}^{1}\right) \#^{(h)} \mathbb{q}_{b}$ follows from Proposition 1 and Proposition 2 of the second section, with $a=s=\left(\varrho-{ }^{\partial} \varrho\right)-\left(\varrho_{\hat{0}^{(i)}}-{ }^{\partial} \varrho_{\hat{0}^{(i j)}}\right)-\left(\varrho_{\hat{2}^{(i j)}}-{ }^{\partial} \varrho_{\hat{2}^{(i j)}}\right)+\varrho_{\hat{0}^{(i j)} \hat{2}^{(i j)}}$, and $b=r-s={ }^{\partial} \varrho+\sum_{k=0}^{3}(-1)^{k} \varrho_{\hat{k}^{(i)}}-{ }^{\partial} \varrho_{\hat{0}^{(i j)}}-{ }^{\partial} \varrho_{\hat{2}^{(i)}}-\varrho_{\hat{0}^{(i)}} \hat{\Upsilon}^{(i j)}+\varrho_{\hat{1}^{(i)} \hat{3}^{(i j)}}$.

In case the crystallization $(\Gamma, \gamma)$ being assumed to gain the regular genus $\mathcal{G}\left(M^{5}\right)$ of the 5-manifold $M^{5}$, then Lemma 6 may be improved as follows.

PROPOSITION 7. Let $M^{5}$ be a connected orientable PL 5-manifold $M^{5}$, with $h \geq 1$ boundary components; let $(\Gamma, \gamma) \in \mathcal{G}_{6}$ be a crystallization of $M^{5}$ and $\varepsilon=\left(\varepsilon_{1}, \ldots \varepsilon_{4}, \varepsilon_{5}=\right.$ 5) be a cyclic permutation of $\Delta_{5}$, so that $\varrho_{\varepsilon}(\Gamma)=\mathcal{G}\left(M^{5}\right)$. If there exist $i, j \in \Delta_{5}$ such that $\varrho_{\hat{i} j}=0$, then $\varrho_{\hat{k}}=0$ for every $k \in \Delta_{5}$, and $M^{5} \cong \#_{\varrho-\partial_{\varrho}}\left(\mathbb{S}^{4} \times \mathbb{S}^{1}\right) \#^{(h)} \mathbb{Z}_{\partial_{\varrho}}$.

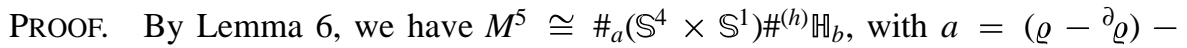
$\left(\varrho_{\hat{0}^{(i)}}-{ }^{\partial} \varrho_{\hat{0}^{(i)}}\right)-\left(\varrho_{\hat{2}^{(i)}}-{ }^{\partial} \varrho_{\hat{2}^{(i j)}}\right)+\varrho_{\hat{0}^{(i)} \hat{2}^{(i)}}$, and $b={ }^{\partial} \varrho+\sum_{k=0}^{3}(-1)^{k} \varrho_{\hat{k}^{(i)}}-{ }^{\partial} \varrho_{\hat{0}^{(i)}}-{ }^{\partial} \varrho_{\hat{2}^{(i j)}}-$ $\varrho_{\hat{0}^{(i)} \hat{\Upsilon}^{(i)}}+\varrho_{\hat{1}^{(i)} \hat{\}}^{(i)}}$. Since the regular genus is sub-additive with respect to connected sum of $n$-manifolds, we have $\varrho=\mathcal{G}\left(M^{5}\right) \leq a+b=\varrho-\varrho_{\hat{1}^{(i)}}-\varrho_{\hat{3}^{(i)}}+\varrho_{\hat{1}^{(i)} \hat{3}^{(i)}} \leq \varrho$, from which $\varrho_{\hat{1}^{(i j)}}=\varrho_{\hat{3}(i)}=0$ obviously follows. Moreover, $\mathcal{G}\left(\partial M^{5}\right)=b={ }^{\partial} \varrho \varrho_{\hat{0}^{(i)}}+\varrho_{\hat{2}^{(i)}}-$ ${ }^{\partial} \varrho_{\hat{0}^{(i)}}-{ }^{\partial} \varrho_{\hat{2}^{(i)}}-\varrho_{\hat{0}^{(i)} \hat{2}^{(i)}} \geq{ }^{\partial}$ implies $\varrho_{\hat{0}^{(i)}}={ }^{\partial} \varrho_{\hat{0}^{(i j)}}, \varrho_{\hat{2}^{(i j)}}={ }^{\partial} \varrho_{\hat{2}^{(i)}}$ and $\varrho_{\hat{0}^{(j)} \hat{2}^{(j)}}=0$. Further, we

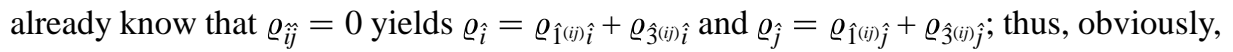
$\varrho_{\hat{i}}=\varrho_{\hat{j}}=0$ follows. Finally, it is now easy to check-by means of formula (7)—that also $\varrho_{\hat{0}_{\hat{(i j)}}}=\varrho_{\hat{2}_{\hat{2}}^{(i)}}=0$ holds, and hence the statement results to be completely proved.

We conclude the paragraph with a further result on the topological structure of the subcomplexes $N(i, j, k)$, which improves a similar one already stated in $\left[\mathrm{C}_{3}\right]$ for contracted triangulations of closed 5-manifolds.

LEMMA 8. Let $(\Gamma, \gamma) \in \mathcal{G}_{6}$ be a crystallization of an orientable PL 5-manifold $M^{5}$, with $h \geq 1$ boundary components. If there exist $i, j, k \in \Delta_{5}$, with $i, j$ non-consecutive in $\Delta_{5}-\{k\}$ and $i, k$ non-consecutive in $\Delta_{5},\{i, j\} \neq\{0,4\},\{i, k\} \neq\{0,4\}$ and $g_{\hat{k}} \leq g_{\hat{j}}$, such that $\varrho_{\hat{k}}-\varrho_{\hat{i} \hat{k}}-\varrho_{\hat{j} \hat{k}}=1$ and $\varrho_{\hat{j}}-\varrho_{\hat{i} \hat{j}}-\varrho_{\hat{k} \hat{j}}=1$, then either $N(i, j, k) \cong \mathbb{Y}_{m}$, with $0 \leq m=\varrho-\varrho_{\hat{i}}-\varrho_{\hat{j}}+\varrho_{\hat{i} \hat{j}}-1$ or $N(i, j, k) \cong N(F)^{\partial} \# \mathbb{Y}_{m}$, where $N(F)$ is the regular neighbourhood of a closed orientable (resp. non orientable) surface of genus $g \geq 0$ (resp. $\tilde{g} \geq 1)$ and $m=\varrho-\varrho_{\hat{i}}-\varrho_{\hat{j}}+\varrho_{\hat{i} j}-2 g$ (resp. $\left.m=\varrho-\varrho_{\hat{i}}-\varrho_{\hat{j}}+\varrho_{\hat{i} j}-\tilde{g}\right)$.

PROOF. By formula (4), the hypotheses give:

$$
\begin{gathered}
g_{\hat{i j k} \hat{k}}=g_{\hat{i} \hat{k}}+g_{\hat{j} \hat{k}}+\left(1-g_{\hat{k}}\right) ; \\
g_{\hat{i j} \hat{k}}=g_{\hat{i} j}+g_{\hat{j} \hat{k}}+\left(1-g_{\hat{j}}\right) ; \\
g_{\hat{i} \hat{k}}=g_{\hat{i} \hat{j}}+g_{\hat{k}}-g_{\hat{j}} .
\end{gathered}
$$


We want now to perform a sequence of elementary collapses on $K(i, j, k)$, not affecting $K(i, j)$ : since it is known that the edges between two fixed vertices are a set of generators for the fundamental group (see [CP]), this implies that, at every step, the total number of edges belonging to $K(i, k) \cup K(j, k)$ which are face of no triangle must be $g_{\hat{k}}-1$ at most. Thus, if $g_{\hat{r} \hat{k}} \leq g_{\hat{s} \hat{k}}$ is assumed, with $\{r, s\}=\{i, j\}$, relation $g_{\hat{i j k} \hat{k}} \leq 2 g_{\hat{s} \hat{k}}-\left(g_{\hat{k}}-1\right)$ yields only the following two possibilities:

(-) exactly $g_{\hat{k}}-1$ edges of $K(s, k)$ are face of no triangle of $K(i, j, k)$, while the other ones are face of exactly two triangles;

(-) some triangles of $K(i, j, k)$ may collapse from their "free" edges belonging to $K(s, k)$.

By iterating the process, a new pseudocomplex $\mathcal{C}$ (consisting of $2 c \geq 0$ triangles) is obtained, so that (if $c \neq 0$ ):

- $C$ contains $c$ edges belonging to $K(i, k)$ and $c$ edges belonging to $K(j, k)$ which are face of exactly two triangles of $\mathcal{C}$, and $g_{\hat{k}}-1$ further edges, belonging to $K(l, k)$ (with $l \in\{i, j\}$ ), which are face of no triangle of $\mathcal{C}$;

- $C$ contains all $g_{\hat{i}}$ edges of $K(i, k)$.

Now, let $\mathcal{S}$, with $\# \mathcal{S}=p \geq 0$, be the (possibly empty) subset of triangles of $\mathcal{C}$, having a "free" edge belonging to $K(i, j)$; it is easy to check that, for every $\sigma \in \mathcal{S}$, the collapse of $\sigma$ allows a finite sequence of collapses of triangles of $\mathcal{C}$, from their "free" edges belonging alternatively to $K(j, k)$ and to $K(i, k)$, till an edge of $K(i, k)$ (which was face of two triangles in $C$ ) results to contain no triangle.

Hence, it is easy to check that the resulting pseudocomplex $C^{\prime}$ consists of $2 c^{\prime}$ triangles $\left(0 \leq c^{\prime} \leq c\right)$ and contains:

- $c^{\prime}$ edges belonging to $K(i, k)$ and $c^{\prime}$ edges belonging to $K(j, k)$ which are face of exactly two triangles of $C^{\prime}$;

- $p$ edges belonging to $K(i, k)$ which are face of no triangle of $C^{\prime}$;

- $g_{\hat{k}}-1$ further edges, belonging to $K(l, k)$ (with $l \in\{i, j\}$ ), which are face of no triangle of $C$;

- $g_{\hat{i} j}-p$ edges belonging to $K(i, j)$.

Moreover, the number of edges belonging to $K(i, j)$ which are face of no triangle in $C^{\prime}$ must be less or equal to the number of edges of $K(i, k)$ which have been "lost" in the process from $K(i, j, k)$ to $C^{\prime}$ (since the edges of $K(i, k)$ are a set of generators for the fundamental group, too); thus, at least $\left(g_{\hat{i} j}-p\right)-\left(g_{\hat{i} \hat{k}}-c^{\prime}-p\right)=c^{\prime}+\left(g_{\hat{j}}-g_{\hat{k}}\right) \geq c^{\prime}$ edges belonging to $K(i, k)$ are face of more than two triangles of $C^{\prime}$.

This obviously implies that either $C^{\prime}$ results to be a graph (from which $N(i, j, k) \cong \mathbb{Y}_{m}$, with $0 \leq m=g_{\hat{i j}}-g_{\hat{i}}-g_{\hat{j}}=\varrho-\varrho_{\hat{i}}-\varrho_{\hat{j}}+\varrho_{\hat{i}}-1$ easily follows) or $C^{\prime}$ may be obtained from the triangulation $T$ of an orientable (resp. non-orientable) surface $F$ of genus $g \geq 0$ (resp. $\tilde{g} \geq 1$ ), by suitable vertices identifications and by further addition of external edges, having the end-points on $T$.

Finally, since each vertex identification and each external edge corresponds to a 1handle attached to the regular neighbourhood of the surface, the thesis follows by a direct computation:

$$
N(i, j, k) \cong N(F)^{\partial} \# \mathbb{Y}_{m}
$$


with $m=\left[\left(2-2 g+c^{\prime}\right)-\left(g_{\hat{i}}+g_{\hat{j}}+1\right)\right]+\left(g_{\hat{i} j}-c^{\prime}\right)=g_{\hat{i} j}-g_{\hat{i}}-g_{\hat{j}}+1-2 g=\varrho-\varrho_{\hat{i}}-\varrho_{\hat{j}}+\varrho_{\hat{i j}}-2 g$ (resp. $\left.m=\left[\left(2-\tilde{g}+c^{\prime}\right)-\left(g_{\hat{i}}+g_{\hat{j}}+1\right)\right]+\left(g_{\hat{j}}-c^{\prime}\right)=g_{\hat{i} j}-g_{\hat{i}}-g_{\hat{j}}+1-\tilde{g}=\varrho^{-}-\varrho_{\hat{i}}-\varrho_{\hat{j}}+\varrho_{\hat{j}}-\tilde{g}\right)$

4. Proofs of the main theorems. We are now able to prove the first of our characterization theorems, which concerns 5-manifolds whose regular genus exceeds the regular genus of their boundary by less than three.

ProOf OF THEOREM 3. Let $M^{5}$ be a connected orientable PL 5-manifold $M^{5}$, with $h \geq 1$ boundary components, satisfying the inequality $\mathcal{G}\left(M^{5}\right) \leq \mathcal{G}\left(\partial M^{5}\right)+2$; further, let $(\Gamma, \gamma) \in \mathcal{G}_{6}$ be a crystallization of $M^{5}$ and $\varepsilon=\left(\varepsilon_{1}, \ldots \varepsilon_{4}, \varepsilon_{5}=5\right)$ be a cyclic permutation of $\Delta_{5}$, so that $\varrho=\varrho_{\varepsilon}(\Gamma)=\mathcal{G}\left(M^{5}\right)$.

Since ${ }^{\partial} \varrho=\varrho_{\varepsilon_{5}}(\partial \Gamma) \geq \mathcal{G}\left(\partial M^{5}\right)$, relation (9) yields:

$$
\left(\varrho_{\hat{0}}-{ }^{\partial} \varrho_{\hat{0}}\right)+\left(\varrho_{\hat{2}}-{ }^{\partial} \varrho_{\hat{2}}\right)+\left(\varrho_{\hat{4}}-{ }^{\partial} \varrho_{\hat{4}}\right) \leq 2\left(\varrho-{ }^{\partial} \varrho\right) \leq 4 ;
$$

hence, there exists $i \in\{0,2,4\}$ so that $\varrho_{\hat{i}}-{ }^{\partial} \varrho_{\hat{i}} \leq 1$.

Now, the inequality $\varrho_{\hat{i}}-{ }^{\partial} \varrho_{\hat{i}}-\varrho_{\hat{i} j}-\varrho_{\hat{i} \hat{k}} \geq 0$ (which holds true for every $j, k$ nonconsecutive in $\Delta_{4}-\{i\}$ ) implies the existence of two colours $i, j \in \Delta_{5}$ such that $\varrho_{i j}=0$. Thus, $M^{5} \cong \#_{\varrho_{-} \partial_{\varrho}}\left(\mathbb{S}^{4} \times \mathbb{S}^{1}\right) \#^{(h)} \mathbb{Z}_{\partial_{\varrho}}$ directly follows from Proposition 7 , and the direct implications of statements (a), (b), (c) result to be proved.

As far as the reversed implications are concerned, it is sufficient to recall that $\mathcal{G}\left(\mathbb{S}^{4} \times \mathbb{S}^{1}\right)=1$ and $\mathcal{G}\left(\mathbb{Y}_{\alpha}\right)=\mathcal{G}\left(\partial \mathbb{Y}_{\alpha}\right)=\alpha$, and to make use both of the sub-additivity of regular genus with respect to connected sum and of relation $\operatorname{rk}\left(\pi_{1}\left(M^{n}\right)\right) \leq \mathcal{G}\left(M^{n}\right)$ (see $[\mathrm{CP}])$.

Let us now assume $(\Gamma, \gamma) \in \mathcal{G}_{6}$ be a crystallization of $M^{5}$, and $\varepsilon=\left(\varepsilon_{1}, \ldots \varepsilon_{4}, \varepsilon_{5}=5\right)$ be a cyclic permutation of $\Delta_{5}$, so that $\varrho=\varrho_{\varepsilon}(\Gamma)=\mathcal{G}\left(M^{5}\right)$ and ${ }^{\partial} \varrho=\varrho_{\varepsilon_{\xi}}(\partial \Gamma)=\varrho-3$. Relation (9) yields:

$$
\left(\varrho_{\hat{0}}-{ }^{\partial} \varrho_{\hat{0}}\right)+\left(\varrho_{\hat{2}}-{ }^{\partial} \varrho_{\hat{2}}\right)+\left(\varrho_{\hat{4}}-{ }^{\partial} \varrho_{\hat{4}}\right) \leq 2\left(\varrho-{ }^{\partial} \varrho\right)=6 ;
$$

hence, either there exists $i \in\{0,2,4\}$ so that $\varrho_{\hat{i}}-{ }^{\partial} \varrho_{\hat{i}} \leq 1$ (which implies $M^{5} \cong$ $\#_{\varrho^{-} \partial_{\varrho}}\left(\mathbb{S}^{4} \times \mathbb{S}^{1}\right) \#^{(h)} \mathbb{Z}_{\partial_{\varrho}}$, as above proved) or $\dot{g}_{\hat{0} \hat{4} \hat{4}}=2 h-2$ and $\varrho_{\hat{i}}-{ }_{\varrho_{\hat{i}}}=2$ for every $i \in\{0,2,4\}$. In the second case, it is not difficult to check that, if $\varrho_{\hat{i} j} \geq 1 \forall i, j \in \Delta_{5}$ is assumed (otherwise, $M^{5} \cong \#_{\varrho^{-} \partial_{\varrho}}\left(\mathbb{S}^{4} \times \mathbb{S}^{1}\right) \#^{(h)} \mathbb{\boxplus}_{\partial_{\varrho}}$ would follow again), then $\varrho_{\hat{i} j}=1$ and $\varrho_{\hat{i} \hat{\zeta}}={ }^{\partial} \varrho_{\hat{i}}=\varrho_{\hat{i}}-2$ hold $\forall i \in\{0,2,4\}, j \neq 5$; moreover, formula (5) implies that relations $\varrho_{\hat{i} \hat{j}}=1$ and $\varrho_{\hat{i} \hat{j}}=\varrho_{\varrho_{\hat{i}}}=\varrho_{\hat{i}}-2$ hold also for $i \in\{1,3\}, \forall j \neq 5$. Hence, $\varrho_{\hat{1}}-\varrho_{\hat{1} \hat{\zeta}}-\varrho_{\hat{1} \hat{S}}=\varrho_{\hat{\zeta}}-$ $\varrho_{\hat{1} \hat{1}}-\varrho_{\hat{3} \hat{\zeta}}=1$ easily follows, and Lemma 8 yields the topological structure of $N(1,3,5)$ :

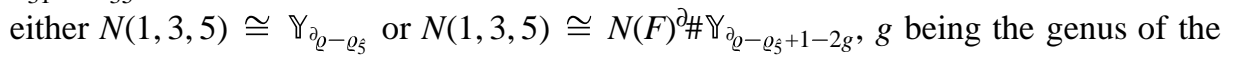
closed surface $F$. On the other hand, since $\varrho_{0}-{ }^{\partial} \varrho_{0}-\varrho_{0 \hat{2}}-\varrho_{0 \hat{4}}=0$, Lemma 5(b) yields $N(0,2,4)=\mathbb{Y}_{s}$, with $s=\dot{g}_{\hat{2}, \hat{4}}-\left(g_{\hat{2}}+g_{\hat{4}}-1\right)+h=\left(\varrho-{ }^{\partial} \varrho\right)-\left(\varrho_{\hat{2}}-\varrho_{\hat{2}}\right)-\left(\varrho_{\hat{4}}-\varrho_{\hat{4}}\right)+\varrho_{\hat{2} \hat{4}}=0$, i.e. $N(0,2,4) \cong \mathbb{D}^{5}$. Finally, since $\pi_{1}\left(M^{5}\right)=\pi_{1}(N(1,3,5))=*_{m} \mathbb{Z}$ implies $F \cong \mathbb{S}^{2}$, the 
thesis follows from Proposition 1 and Proposition 2 of the second section (in the partic-

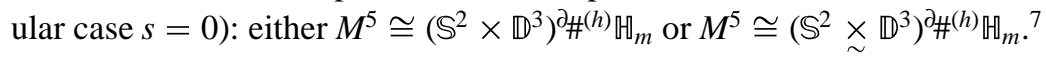

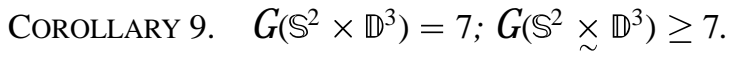

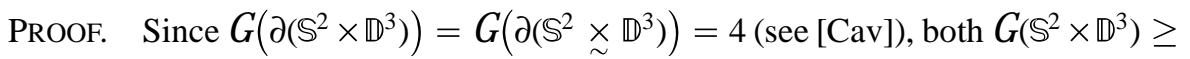
7 and $\mathcal{G}\left(\mathbb{S}^{2} \underset{\sim}{\times \mathbb{D}^{3}}\right) \geq 7$ are direct consequences of Theorem $3(\mathrm{a})$,(b).

On the other hand, a crystallization of $\mathbb{S}^{2} \times \mathbb{D}^{3}$ of genus seven may be easily obtained from the 6-coloured graph $\left(\Gamma^{(1)}, \gamma^{(1)}\right)$ depicted in Figure 1, which represents $\mathbb{S}^{2} \times \mathbb{D}^{3}$ and satisfies $\varrho_{\varepsilon}\left(\Gamma^{(1)}\right)=7, \varepsilon$ being the identity on $\Delta_{5}:\left(\Gamma^{(1)}, \gamma^{(1)}\right)$ directly results from the "standard" crystallizations of $\mathbb{S}^{2}$ and $\mathbb{D}^{3}$, by an obvious modification of the construction described in [GG].

Proof OF THEOREM 4. As usual, if $M^{5}$ is a connected orientable PL 5-manifold $M^{5}$, with $h \geq 1$ boundary components, let $(\Gamma, \gamma) \in \mathcal{G}_{6}$ be a crystallization of $M^{5}$ and $\varepsilon=\left(\varepsilon_{1}, \ldots \varepsilon_{4}, \varepsilon_{5}=5\right)$ be a cyclic permutation of $\Delta_{5}$, so that $\varrho=\varrho_{\varepsilon}(\Gamma)=\mathcal{G}\left(M^{5}\right)$.

It is known that, for every choice of $\{i, j\} \in \Delta_{5}$, a presentation $\langle X ; R\rangle$ for the fundamental group $\pi_{1}\left(M^{5}\right)$ of $M^{5}$ exists, where $X$ is the set of all $\left(\Delta_{5}-\{i, j\}\right)$-residues of $(\Gamma, \gamma)$, but $g_{\hat{i}}+g_{\hat{j}}-1$ arbitrarily chosen (see [CP]); thus, formula (4) implies that

$$
\operatorname{rk}\left(\pi_{1}\left(M^{5}\right)\right) \leq \varrho-\varrho_{\hat{i}}-\varrho_{\hat{j}}+\varrho_{\hat{j}}
$$

for every $i, j$ non-consecutive in $\Delta_{5},\{i, j\} \neq\{0,4\}$.

Now, if $\mathcal{G}\left(M^{5}\right)=\varrho=\operatorname{rk}\left(\pi_{1}\left(M^{5}\right)\right)$ is assumed, $\varrho_{\hat{i}}=0$ obviously holds, for every $i \in \Delta_{5} ;$ so, $M^{5} \cong \#_{\varrho_{-} \partial_{\varrho}}\left(\mathbb{S}^{4} \times \mathbb{S}^{1}\right) \#^{(h)} \mathbb{Z}_{\partial_{\varrho}}$ directly follows from Proposition 7 .

On the other hand, the inequality $\varrho_{\hat{i}}+\varrho_{\hat{j}}-\varrho_{\hat{j} j} \leq \varrho-\operatorname{rk}\left(\pi_{1}\left(M^{5}\right)\right)$, together with relation (7), yields

$$
\sum_{k \neq i, j}\left(\varrho_{\hat{i} \hat{k}}+\varrho_{\hat{j} \hat{k}}\right) \leq \sum_{k \neq i, j}\left(\varrho_{\hat{i} \hat{k}}+\varrho_{\hat{j} \hat{k}}\right)+{ }^{\partial} \varrho_{\hat{i}}+{ }^{\partial} \varrho_{\hat{j}} \leq 2\left(\varrho-\operatorname{rk}\left(\pi_{1}\left(M^{5}\right)\right)\right)
$$

for every $i, j$ non-consecutive in $\Delta_{5},\{i, j\} \neq\{0,4\}$.

Now, if $\mathcal{G}\left(M^{5}\right)-\operatorname{rk}\left(\pi_{1}\left(M^{5}\right)\right) \leq 4$ is assumed, the existence of at least an addendum $\varrho_{\hat{r} \hat{s}}=0$ easily follows: in fact, there are eight non-negative addenda in the first member of each inequality, and they can not be all equal to one, since relation (8) gives $\sum_{j \neq 5} \varrho_{5 \hat{j}}=$ $2 \varrho_{\hat{s}} \in \mathbb{P}$.

Thus, $M^{5} \cong \#_{\varrho^{-\partial_{\varrho}}}\left(\mathbb{S}^{4} \times \mathbb{S}^{1}\right) \#^{(h)} \mathbb{H}_{\partial_{\varrho}}$ again follows from Proposition 7 .

As far as parts (c) and (d) is concerned, note that the Euler characteristic computation for closed 4-manifolds (see $\left[\mathrm{C}_{1}\right]$ or $[\mathrm{Cav}]$ ), applied to each connected component of $\partial M^{5}$,

\footnotetext{
7 Note that $N\left(\mathbb{S}^{2}\right)$ is surely PL-homeomorphic to one or another of the two 3-ball bundles over the 2-sphere (see [B]), and that the case $N(1,3,5) \cong \mathbb{Y}_{\partial_{\varrho-\varrho_{5}}}$ implies $M^{5} \cong{ }^{(h)} \mathbb{U}_{\partial_{\varrho-} \varrho_{\hat{\xi}}}$, which contradicts the hypothesis $\mathcal{G}\left(M^{5}\right)={ }^{\partial} \varrho+3$.
} 


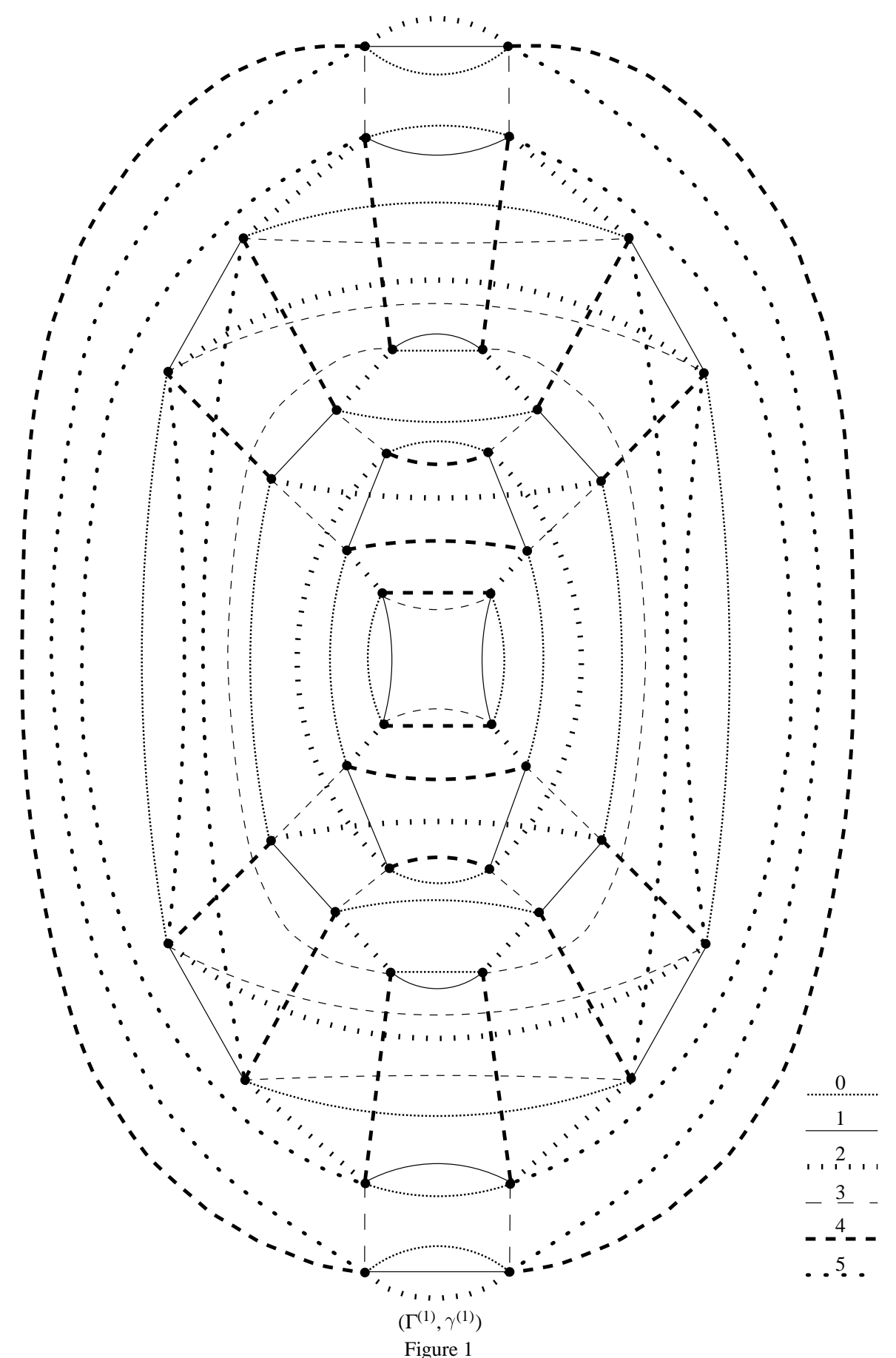


gives:

$$
\begin{aligned}
\beta_{2}\left(\partial M^{5}\right)= & 2 \beta_{1}\left(\partial M^{5}\right)-2^{\partial} \varrho+\sum_{j=0}^{4}{ }^{\partial} \varrho_{\hat{j}} \\
= & {\left[\beta_{1}\left(\partial M^{5}\right)-\left({ }^{\partial} \varrho-{ }^{\partial} \varrho_{\hat{0}^{(i)}}-{ }^{\partial} \varrho_{\hat{2}^{(i)}}\right)\right] } \\
& +\left[\beta_{1}\left(\partial M^{5}\right)-\left({ }^{\partial} \varrho-{ }^{\partial} \varrho_{\hat{1}^{(i)}}-{ }^{\partial} \varrho_{\hat{\zeta}^{(i)}}\right)\right]+{ }^{\partial} \varrho_{\hat{i}} \leq{ }^{\partial} \varrho_{\hat{i}}
\end{aligned}
$$

where, for every $i \in \Delta_{4},\left(0^{(i)}, 1^{(i)}, 2^{(i)}, 3^{(i)}\right)$ denotes the (ordered) 4-tuples induced by $\varepsilon$ on $\Delta_{4}-\{i\}$.

Hence, relation (11) yields

$$
\sum_{k \neq i, j}\left(\varrho_{\hat{i} \hat{k}}+\varrho_{\hat{j} \hat{k}}\right)+2 \beta_{2}\left(\partial M^{5}\right) \leq \sum_{k \neq i, j}\left(\varrho_{\hat{i} \hat{k}}+\varrho_{\hat{j} \hat{k}}\right)+{ }^{\partial} \varrho_{\hat{i}}+{ }^{\partial} \varrho_{\hat{j}} \leq 2\left(\varrho-\operatorname{rk}\left(\pi_{1}\left(M^{5}\right)\right)\right) .
$$

Since no addendum of type $\varrho_{\hat{s}}$ may be equal to zero (otherwise, $M^{5} \cong$ $\#_{\varrho^{-} \partial_{\varrho}}\left(\mathbb{S}^{4} \times \mathbb{S}^{1}\right) \#^{(h)} \mathbb{H}_{\partial_{\varrho}}$ would follow, against the hypothesis) and since the addenda of type $\varrho_{\hat{r} \hat{s}}$ can not be all equal to one (as previously stated), $\varrho-\operatorname{rk}\left(\pi_{1}\left(M^{5}\right)\right) \geq 5+\beta_{2}\left(\partial M^{5}\right)$ easily follows. In particular, $\varrho-\operatorname{rk}\left(\pi_{1}\left(M^{5}\right)\right)=5$ implies either the existence of an addendum $\varrho_{\hat{r} \hat{s}}=0$ (from which $\partial M^{5}=\partial\left(\#_{\varrho_{-\partial_{\varrho}}}\left(\mathbb{S}^{4} \times \mathbb{S}^{1}\right) \#^{(h)} \mathbb{⿴}_{\partial_{\varrho}}\right) \cong \amalg\left\{\#_{\alpha_{i}}\left(\mathbb{S}^{3} \times \mathbb{S}^{1}\right) / i=\right.$ $1, \ldots, h\}$, with $\sum_{i=1}^{h} \alpha_{i}=\partial_{\varrho}$ directly follows) or the existence of ${ }^{\partial} \varrho_{j}=0$ (from which $\partial M^{5} \cong \amalg\left\{\#_{\alpha_{i}}\left(\mathbb{S}^{3} \times \mathbb{S}^{1}\right) / i=1, \ldots, h\right\}$, with $\sum_{i=1}^{h} \alpha_{i}=\alpha=\mathcal{G}\left(\partial M^{5}\right) \leq \partial^{\partial}$, follows by [CM; Lemma 3(a)]).

Now, in order to complete the proof of Theorem 4, we have to prove the reversed implication of part (a); for, it is sufficient to recall that $\mathcal{G}\left(\mathbb{S}^{4} \times \mathbb{S}^{1}\right)=1$ and $\mathcal{G}\left(\mathbb{Y}_{\alpha}\right)=$ $\mathcal{G}\left(\partial \mathbb{Y}_{\alpha}\right)=\alpha$, and to make use both of the sub-additivity of regular genus with respect to connected sum and of relation $\operatorname{rk}\left(\pi_{1}\left(M^{n}\right)\right) \leq \mathcal{G}\left(M^{n}\right)$ (see [CP]).

PROOF OF THEOREM 2. Let $M^{5}$ be a connected orientable PL 5-manifold $M^{5}$, with $h \geq 1$ boundary components, satisfying the inequality $\mathcal{G}\left(M^{5}\right) \leq 5$; further, let $(\Gamma, \gamma) \in$ $\mathcal{G}_{6}$ be a crystallization of $M^{5}$ and $\varepsilon=\left(\varepsilon_{1}, \ldots \varepsilon_{4}, \varepsilon_{5}=5\right)$ be a cyclic permutation of $\Delta_{5}$, so that $\varrho=\varrho_{\varepsilon}(\Gamma)=\mathcal{G}\left(M^{5}\right) \leq 5$.

By Theorem 4(a), (b), the fundamental group of the manifold satisfies either $\operatorname{rk}\left(\pi_{1}\left(M^{5}\right)\right)=\varrho\left(\right.$ which implies $\left.M^{5} \cong \#_{\varrho-\partial_{\varrho}}\left(\mathbb{S}^{4} \times \mathbb{S}^{1}\right) \#^{(h)} \mathbb{\boxplus}_{\partial_{\varrho}}\right)$, or $\operatorname{rk}\left(\pi_{1}\left(M^{5}\right)\right) \leq \varrho-5$ (i.e. $M^{5}$ simply connected with $\varrho=\mathcal{G}\left(M^{5}\right)=5$ ).

Further, Theorem 3 states that the only simply connected 5-manifold $M^{5}\left(M^{5} \neq \#_{h} \mathbb{D}^{5}\right)$

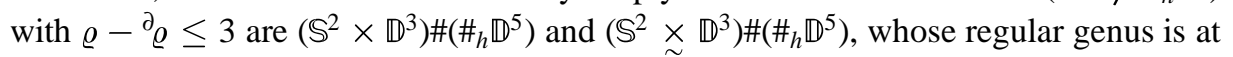
least seven (see Corollary 9); thus, if $\varrho=5$ is assumed, ${ }^{\partial} \varrho \leq 1$ necessarily holds.

Then, by applying relation (4) to each connected component of the boundary graph $\left(\partial \Gamma,{ }^{\partial} \gamma\right)$, the existence of a colour $i \in \Delta_{4}$ so that ${ }^{\partial} \varrho_{\hat{i}}=0$ directly follows; further, [CM; Lemma 3(a)] ensures that $\partial M^{5} \cong \amalg\left\{\#_{\alpha_{i}}\left(\mathbb{S}^{3} \times \mathbb{S}^{1}\right) / i=1, \ldots, h\right\}$, with $\sum_{i=1}^{h} \alpha_{i}=\alpha=$ ${ }^{\partial} \varrho-{ }^{\partial} \varrho_{\hat{O}^{(i)}}-{ }^{\partial} \varrho_{\hat{2}^{(i)}}={ }^{\partial} \varrho-{ }^{\partial} \varrho_{\hat{1}^{(i)}}-{ }^{\partial} \varrho_{\hat{\beta}^{(i)}} \leq 1$.

Now, $\alpha=0$ contradicts the hypothesis of simply connectedness: in fact, $\left[\mathrm{FG}_{2}\right]$ implies $M^{5} \cong \bar{M}^{5} \#\left(\#_{h} \mathbb{D}^{5}\right), \bar{M}^{5}$ being a closed orientable 5-manifold, with $\mathcal{G}\left(\bar{M}^{5}\right)=\mathcal{G}\left(M^{5}\right)=5$, and Theorem 1(b) ensures $\bar{M}^{5} \cong \#_{5}\left(\mathbb{S}^{4} \times \mathbb{S}^{1}\right)$. On the other hand, if $\alpha=1={ }_{\varrho} \varrho$ is 


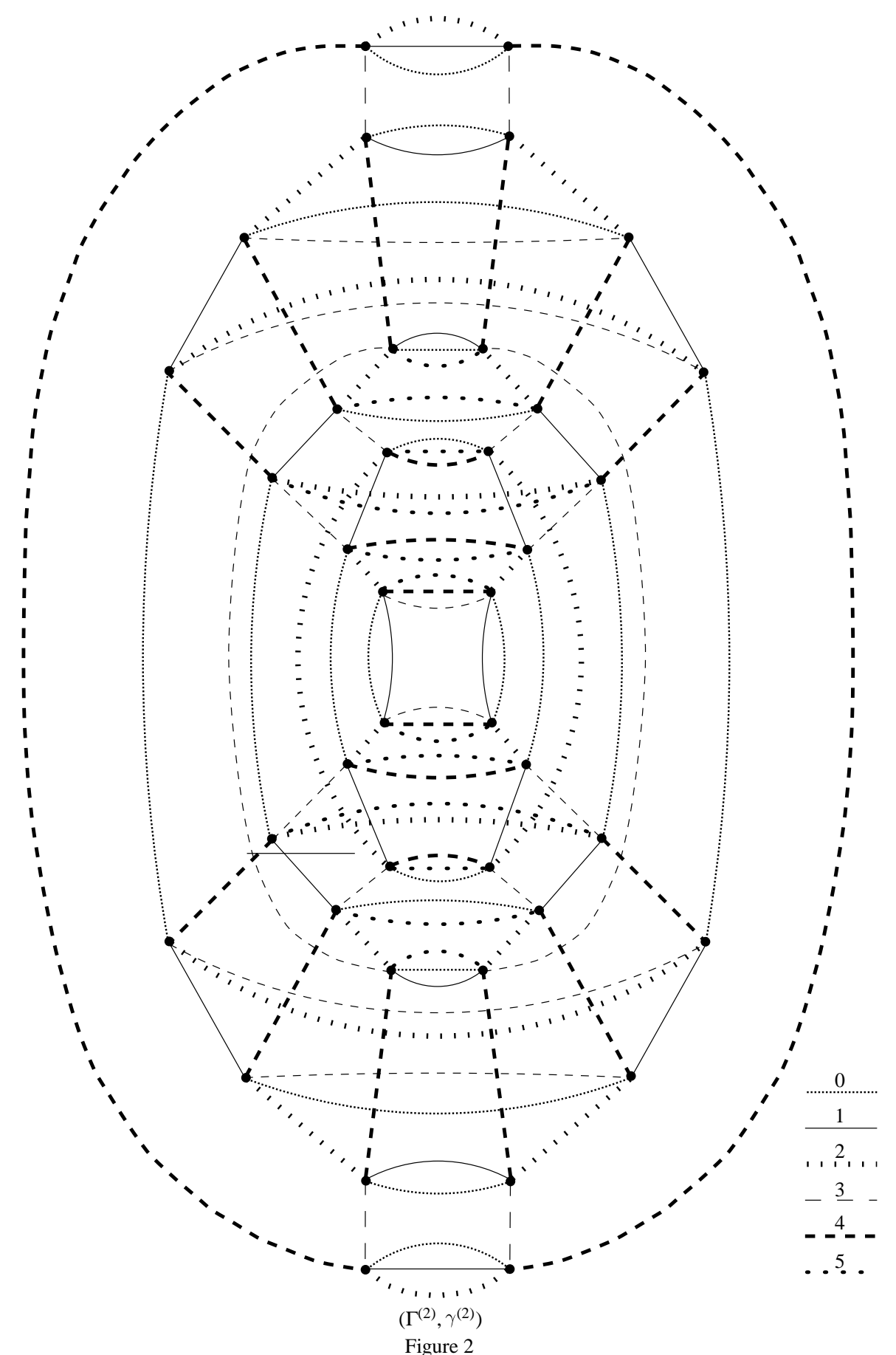


assumed, ${ }^{\partial} \varrho_{\hat{j}}=0$ holds for every $j \in \Delta_{4}$; hence, formula (9) yields $\varrho_{\hat{0}}+\varrho_{\hat{2}}+\varrho_{\hat{4}} \leq$ $2\left(\varrho-{ }^{\partial} \varrho\right)=8$, from which the existence of $i, j \in \Delta_{5}$ so that $\varrho_{\hat{i j}}=0$ easily follows. Hence, another contradiction occurs, since Proposition 7 would imply $M^{5} \cong \#_{4}\left(\mathbb{S}^{4} \times \mathbb{S}^{1}\right) \#^{(h)} \mathbb{W}_{1}$, which is not a simply connected manifold.

COROLlary 10. $\mathcal{G}\left(\mathbb{S}^{3} \times \mathbb{D}^{2}\right)=6$.

Proof. The inequality $\mathcal{G}\left(\mathbb{S}^{3} \times \mathbb{D}^{2}\right) \geq 6$ is a direct consequence of the complete classification of 5-manifolds up to regular genus five (see Theorem 2).

On the other hand, a crystallization of $\mathbb{S}^{3} \times \mathbb{D}^{2}$ of genus six may be easily obtained from the 6-coloured graph $\left(\Gamma^{(2)}, \gamma^{(2)}\right)$ depicted in Figure 2, which represents $\mathbb{S}^{3} \times \mathbb{D}^{2}$ and satisfies $\varrho_{\varepsilon}\left(\Gamma^{(2)}\right)=6, \varepsilon$ being the identity on $\Delta_{5}:\left(\Gamma^{(2)}, \gamma^{(2)}\right)$ directly results from the "standard" crystallizations of $\mathbb{S}^{3}$ and $\mathbb{D}^{2}$, by an obvious modification of the construction described in [GG].

\section{REFERENCES}

[B] D. Barden, Simply connected five-manifolds, Ann. of Math. 82(1965), 365-385.

[C $\mathbf{C}_{1}$ ] M. R. Casali, A combinatorial characterization of 4-dimensional handlebodies, Forum Math. 4(1992), $123-134$.

$\left[\mathbf{C}_{2}\right] \longrightarrow$ A note on the characterization of handlebodies, Europ. J. Combinatorics 14(1993), 301-310.

$\left[\mathbf{C}_{3}\right] \longrightarrow$, On the regular genus of 5-manifolds with free fundamental group, to appear.

[CG] M. R. Casali and C. Gagliardi, Classifying PL 5-manifolds up to regular genus seven, Proc. Amer. Math. Soc. (1)120(1994), 275-283.

[CM] M. R. Casali and L. Malagoli, Handle-decompositions of PL4-manifolds, Cahiers de Topologie et Geom. Diff. Cat., to appear.

[Cav] A. Cavicchioli, On the genus of smooth 4-manifolds, Trans. Amer. Math. Soc. 31(1992), 203-214.

[CH] A. Cavicchioli and F. Hegenbarth, On the determination of PL-manifolds by handles of lower dimension, Topology Appl. (2)53 (1993), 111-118.

[CP] R. Chiavacci and G. Pareschi, Some bounds for the regular genus of PL-manifolds, Discrete Math. 82(1990), 165-180.

[Co] A. Costa, Coloured graphs representing manifolds and universal maps, Geometriae Dedicata 28(1988), 349-357.

[FG $\left.{ }_{1}\right]$ M. Ferri and C. Gagliardi, The only genus zero n-manifold is $\mathbb{S}^{n}$, Proc. Amer. Math. Soc. 85(1982), $638-642$.

[FG $\left.\mathbf{F G}_{2}\right]$ _ A characterization of punctured n-spheres, Yokohama Math. J. 33(1985), 29-38.

[FGG] M. Ferri, C. Gagliardi and L. Grasselli, A graph-theoretical representation of PL-manifolds. A survey on crystallizations, Aequationes Math. 31(1986), 121-141.

$\left[\mathbf{G}_{1}\right]$ C. Gagliardi, Extending the concept of genus to dimension n, Proc. Amer. Math. Soc. 81(1981), 473-481.

$\left[\mathbf{G}_{2}\right] \ldots$, Regular genus: the boundary case, Geometriae Dedicata 22(1987), 261-281.

[GG] C. Gagliardi and L. Grasselli, Representing products of polyhedra by products of edge-colored graphs, Journal of Graph Theory (5)15(1993), 549-579.

[HW] P. J. Hilton and S. Wylie, An introduction to algebraic topology - Homology theory, Cambridge Univ. Press, Cambridge, 1960.

[L] F. Laudenbach, Topologie de la dimension trois: homotopie et isotopie, Soc. Math. de France, Asterisque 12, 1974.

[LP] F. Laudenbach and V. Poenaru, A note on 4-dimensional handlebodies, Bull. Soc. Math. France 100 (1972), 337-344.

[LM] S. Lins and A. Mandel, Graph-encoded 3-manifolds, Discrete Math. 57(1985), 261-284.

[M] J. M. Montesinos, Heegaard diagrams for closed 4-manifolds, Geometric Topology (J. Cantrell Ed.), Proc. 1977 Georgia Conference, Academic Press, 1979, 219-237. 
[P] M. Pezzana, Sulla struttura topologica delle varietà compatte, Atti Sem. Mat. Fis. Univ. Modena 23(1974), 269-277.

[RS] C. P. Rourke and B. J. Sanderson, Introduction to piecewise-linear topology, Springer-Verlag, BerlinHeidelberg-New York, 1972.

[V] A. Vince, $n$-graph, Discrete Math. 72(1988), 367-380.

Dipartimento di Matematica

Via Campi 213 B

I-41100 MODENA

Italy

e-mail: casali@unimo.it 